
Hui*

\title{
Influence of different material parameters on nonlinear vibration of the cylindrical skeleton supported prestressed fabric composite membrane
}

https://doi.org/10.1515/rams-2021-0026

Received Jan 24, 2021; accepted Feb 22, 2021

\section{Introduction}

Membrane structure, also known as fabric structure, is a flexible large-span spatial structure developed in the middle of the century [1]. It has the characteristics of light weight, large span, good seismic performance, convenient construction and good self-cleaning. Steel skeleton supported membrane structure is the main structure of membrane structure, accounting for more than $50 \%$ of all membrane structure engineering. Compared with the traditional rigid structure, the structural characteristics of the membrane structure determine that it is essential to analyze the shape-finding and self-vibration characteristics during the design process [2]. At present, in the vibration analysis of cylindrical skeleton supported prestressed fabric composite membrane, there are few studies that consider both the geometric nonlinearity of membrane deformation and the influence of different parameters of membrane material on membrane vibration.

In recent years, the dynamic response of cylindrical shell structure has received more and more attentions, and more researches have been done on the nonlinear vibration of membrane structures. By studying the effects of initial geometric imperfections and boundary conditions on the linear and nonlinear vibration behaviors of simply supported circular plates, Hui $[3,4]$ found that with the increase of linear vibration frequency, the obvious soft spring characteristics appeared in the nonlinear vibration. After that, he also studied the Runge-Kutta numerical solutions

^Corresponding Author: David Hui: Department of Mechanical Engineering, University of New Orleans, New Orleans, LA 70148, United States of America; Email: DHui@uno.edu

Zhoulian Zheng: School of Civil Engineering, Chongqing University, Chongqing, 400045, China

Jian Liu: School of Civil Engineering, Guangzhou University, Guangzhou 510006, China

Haibing Xie, Su Jiang: College of Environment and Civil Engineering, Chengdu University of Technology, Chengdu 610059, China

\footnotetext{
*Corresponding Author: Changjiang Liu: School of Civil Engineering, Guangzhou University, Guangzhou 510006, China; Email: *Corresponding Author: Mengfei Wang: College of Environment and Civil Engineering, Chengdu University of Technology, Chengdu 610059, China; Email: wangmengfei@stu.cdut.edu.cn
}

O Open Access. (c) 2021 C. Liu et al., published by De Gruyter. (Cc) BY License
This work is licensed under the Creative Commons Attribution 4.0 
of the modified Duffing ordinary differential equations for simply supported rectangular plates and shallow spherical shells, and found that the soft spring characteristic of the main chain curve was effective when the amplitude was small. Awrejcewicz et al. [5] and Krysko et al. [6] proposed a method to study the free vibration of a rectangular nonuniform shell, and analyzed the nonlinear characteristics of a flat elastic rectangular spherical panel under uniformly distributed transverse load. Tang and Zhao [7] studied the spatial and temporal directions of a multi symplectic integrator by using the Multi Symplectic Runge Kutta Nystrom method, and obtained the membrane free vibration equation. Xu et al. [8, 9] applied a large amplitude theory to study the aerodynamic stability of a geometric nonlinear anisotropy hyperbolic parabolic tensioned tensile structure. Liu et al. [10, 11] studied the nonlinear instability of shallow shells under uniform load and circular line load, which provided a theoretical basis for engineering design and instability prediction and control of shallow shells. Zheng et al. [12] proposed a nonlinear free vibration analysis method for axisymmetric polar anisotropy circular membranes based on the large deflection theory and virtual displacement principle. Gupta et al. [13] established a nonlinear vibration analysis model for a partially cracked thin anisotropy thin plate affected by fiber orientation and partial cracks. Sofiyev et al. [14] and Sofiyev and Kuruoglu [15] studied the nonlinear free vibration behavior of functionally graded anisotropy cylindrical shells interacting with two-parameter elastic foundations. At the same time, according to the shear deformation theory (SDT), the nonlinear dynamic response of a heterogeneous anisotropic cylinder on a nonlinear elastic foundation was studied. Li et al. $[16,17]$ applied the von Karman large deformation theory to establish the stochastic motion equation of the membrane structure, and obtained the stochastic dynamic response result by perturbation method. At the same time, the theoretical analysis of the free vibration of anisotropy rectangular membrane structures was carried out on the basis of considering the coupling effect between modes. Since it was not clear how much the vibration of flexible attachments would affect their normal functions or spacecraft control, Oberst and Tuttle [18] conducted experimental verification on the dynamic and stability behavior of thinwalled structures. Tang et al. [19] analyzed the effects of initial tension ratio, pressure and voltage on the nonlinear free vibration of spherical dielectric elastomer balloons. Wu et al. [20] and Shao et al. [21-24] studied the geometrically nonlinear large deflection vibration of a moving membrane with variable speed by using Galerkin method and thin plate theory. Based on the geometric nonlinear vibration of rectangular anisotropy membrane structure, Liu et al. [25] proposed a nondestructive testing method to monitor its preloading force. Wang et al. [26] studied the nonlinear dynamic characteristics of the microplate system under the action of electrostatic force on both sides.

The perturbation method, also known as the small parameter expansion method, is an effective mathematical method for solving the approximate solution of nonlinear problems. This method is widely used in many mechanical and practical engineering problems. Awrejcewicz and Dzyubak [27] applied the perturbation analysis method to study the two degree of freedom nonlinear dynamics of the rotor supported by the magnetohydrodynamic bearing. Stepanova and Igonin [28] used perturbation method to solve the nonlinear eigenvalue problem caused by fatigue crack growth. It was shown that the perturbation method based on small parameters could quantitatively describe the closeness between the eigenvalues of the perturbed nonlinear problems and the eigenvalues of the linear undisturbed linear problems, and obtained the closed form solution accordingly. Yazdi [29] studied the nonlinear forced vibration of anisotropy circular plates on an elastic foundation by Galerkin method and homotopy perturbation method. Zhu et al. [30] investigated the effect of surface energy on the nonlinear free vibration behavior of anisotropy piezoelectric cylindrical shells through Hamiltonian principle and homotopy perturbation method. Based on von Karman's large deflection theory, Bubov-Galerkin method and KBM perturbation method, Liu et al. [31, 32] studied the theory and numerical application of nonlinear forced vibration of anisotropy rectangular membrane and saddle membrane structures under impact load. Singh and Sharma [33] used the method of homotopy perturbation combined with Laplace transform to solve nonlinear fractional differential equations.

In recent years, the research on the manufacture and properties of composite fabric materials such as membrane materials emerge in an endless stream. Dong et al. [34] proposed an effective method to study the mechanical properties of composite fabric rubber composites at different temperatures. Bolcu and Stanescu [35] studied the mechanical properties of composite fabric reinforcement from the aspects of characteristic curve, elastic modulus, tensile strength, elongation at break, specific frequency and damping factor. Lofy et al. [36] studied the polarization rotation of light propagating in left-handed thin membrane and layered structures. Large resonance enhancement of reflection Kerr angle could be obtained in left-handed thin membranes through experiments. Wang et al. [37] grew $\mathrm{Cu}_{2} \mathrm{O}$ thin membranes through different ways, such as autooxidation and metal-organic deposition, and studied the surface morphology and chemical states of the membranes 
under different reduction conditions. Chang [38] studied the thermo-optical properties and oxygen transmittance of colorless and transparent polyimide (CPI) membranes at different biaxial tensile ratios, and its performance was compared and analyzed. Shi et al. [39] studied the true biaxial properties (stress-strain properties and fracture criteria) of typical polyvinylidene fluoride (PVDF) coated polyester membrane materials. Li et al. [40-43] used the Jacobi-Ritz method to analyze the free vibration problems of composite laminated doubly-curved shells of revolution with general boundary conditions and laminated composite shallow shells with arbitrary boundary conditions. They also studied the free vibration behavior of composite laminated cylindrical shells under complex and arbitrary boundary conditions by semi-analytical method, and verified the spring stiffness, Jacobian and other parameters numerically.

The nonlinear equation cannot be solved directly, but the perturbation method can solve the nonlinear equation better so as to get its approximate analytical solution. This paper adopted the KBM perturbation method in combination with Galerkin method to solve the nonlinear vibration partial differential control equation of damped prestressed nonlinear vibration of large deflection and asymmetric membrane structures. By comparing the analytical solution of the KBM perturbation method with the numerical solution of the Runge-Kutta method, the effective range of the analytical solution was determined. Finally, the effects of different membrane prestress, different length width ratio, different rise span ratio and different membrane material parameters on the nonlinear free vibration of prestressed fabric composite membrane with cylindrical skeleton were analyzed by numerical examples, and some rules were obtained.

\section{Theoretical solution process}

\subsection{Surface equation}

The structural model studied in this paper is cylindrical skeleton supported membrane structure as shown in Figure 1. The membrane material is anisotropy, and the boundary condition is four edges clamped. The orthogonal direction $x$ and $y$ are two main fiber directions with different Young's modulus. $a$ and $b$ represent the lengths of the membranes in the $x$ and $y$ directions, respectively. $N_{o x}$ and $N_{o y}$ represent the pretension in $x$ and $y$ directions, respectively. $\kappa_{2}$ represents the mid-span arch in $x$ direction and $\kappa_{1}$ represents the mid-span sag in $y$ direction. Point $\mathrm{A}$ is the center of the membrane structure.

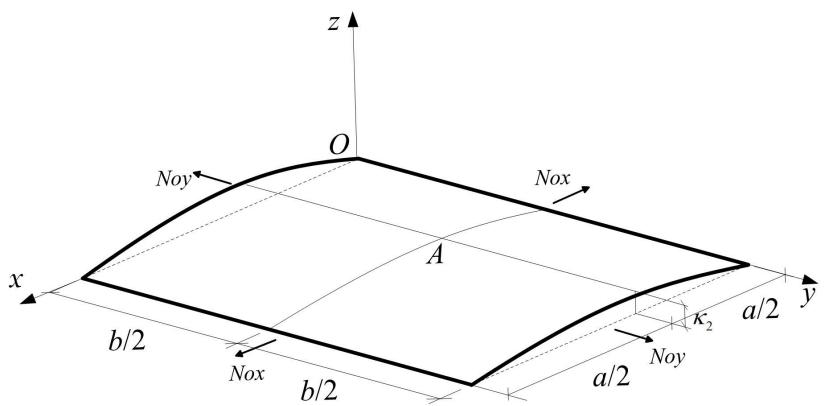

Figure 1: Skeleton supported membrane model with four edges clamped

According to the basic theory of plate and shell [44], the principal curvature in $x$ and $y$ directions can be obtained as follow

$$
\left\{\begin{array}{l}
k_{0 x}=-\frac{\partial^{2} z_{0}}{\partial x^{2}}=\frac{8 \kappa_{2}}{a^{2}} \\
k_{0 y}=\frac{\partial^{2} z_{0}}{\partial y^{2}}=\frac{8 \kappa_{1}}{b^{2}}
\end{array}\right.
$$

Under the action of initial stresses $N_{0 x}$ and $N_{0 y}$ [45], we can obtain

$$
k_{0 x} N_{0 x}+k_{0 y} N_{0 y}=0
$$

Taking $\kappa_{1}=0$ and $k_{0 y}=0$, the curved surface equation of skeleton supported membrane can be obtained as follows

$$
z_{0}(x, y)=-\frac{\kappa_{2}(x-(a / 2))^{2}}{(a / 2)^{2}}
$$

where $z_{0}$ is the initial surface function.

\subsection{Governing equations and boundary conditions}

According to the von Kármán's large amplitude theory and D'Alembert's principle [46-48], the dynamic motion equation and compatible equation of the skeleton supported membrane are given

$$
\left\{\begin{array}{l}
\rho_{0} \frac{\partial^{2} w}{\partial t^{2}}+c \frac{\partial w}{\partial t}-\left(N_{x}+N_{0 x} k_{x}\right. \\
-\left(N_{y}+N_{0 y}\right) k_{y}+2 N_{x y} k_{x y}=0 \\
\frac{1}{E_{1} h} \frac{\partial^{2} N_{x}}{\partial y^{2}}+\frac{1}{E_{2} h} \frac{\partial^{2} N_{y}}{\partial x^{2}}-\frac{\mu_{1}}{E_{1} h} \frac{\partial^{2} N_{x}}{\partial x^{2}}-\frac{\mu_{2}}{E_{2} h} \frac{\partial^{2} N_{y}}{\partial y^{2}} \\
-\frac{1}{G h} \frac{\partial^{2} N_{x y}}{\partial x \partial y}=\left(\frac{\partial^{2} w}{\partial x \partial y}\right)^{2}-\frac{\partial^{2} w}{\partial x^{2}} \frac{\partial^{2} w}{\partial y^{2}}-k_{0 x} \frac{\partial^{2} w}{\partial y^{2}}
\end{array}\right.
$$

where $\rho_{0}$ represents the surface density of membrane material, $c$ represents the damping coefficient. $N_{x}$ and $N_{y}$ represent tension forces in the $x$ and $y$ directions, respectively. $N_{0 x}$ and $N_{0 y}$ represent pretensions in the $x$ and $y$ directions, respectively. $N_{x y}$ represents shear force, $w$ represents deflection $w(x, y, t), h$ represents the thickness of the membrane 
material. $E_{1}$ and $E_{2}$ denote Young's modulus in $x$ and $y$ direction. $\mu_{1}$ and $\mu_{2}$ denote Poisson's ratio in $x$ and $y$ directions. $k_{x}$ and $k_{y}$ denote principal curvatures in $x$ and $y$ directions, respectively. $k_{0 x}$ and $k_{0 y}$ denote initial principal curvatures in $x$ and $y$ directions.

Introduce the stress function $F=F(x, y, t)$ yields [38]

$$
N_{x}=h \frac{\partial^{2} F}{\partial y^{2}}, N_{y}=h \frac{\partial^{2} F}{\partial x^{2}}, N_{x y}=-h \frac{\partial^{2} F}{\partial x \partial y}
$$

Since the shear stress has little influence on the vibration of the membrane, the shear force can be considered as $N_{x y}=0$ and substituted into Eq. (5).

$$
\frac{\partial^{2} N_{x}}{\partial x^{2}}=\frac{\partial^{2} N_{y}}{\partial y^{2}}=h \frac{\partial^{4} F}{\partial x^{2} \partial y^{2}}=-\frac{\partial^{2} N_{x y}}{\partial x \partial y}=0
$$

Substitute Eq. (5) and Eq. (6) into Eq. (4), it can be obtained

$$
\begin{gathered}
\rho_{0} \frac{\partial^{2} w}{\partial t^{2}}+c \frac{\partial w}{\partial t}-\left(h \frac{\partial^{2} F}{\partial y^{2}}+N_{0 x}\right) \frac{\partial^{2} w}{\partial x^{2}} \\
-k_{0 x} h \frac{\partial^{2} F}{\partial y^{2}}-\left(h \frac{\partial^{2} F}{\partial x^{2}}+N_{0 y}\right) \frac{\partial^{2} w}{\partial y^{2}}=0 \\
\frac{1}{E_{1}} \frac{\partial^{4} F}{\partial y^{4}}+\frac{1}{E_{2}} \frac{\partial^{4} F}{\partial x^{4}}=\left(\frac{\partial^{2} w}{\partial x \partial y}\right)^{2}-\frac{\partial^{2} w}{\partial x^{2}} \frac{\partial^{2} w}{\partial y^{2}} \\
-k_{0 x} \frac{\partial^{2} w}{\partial y^{2}}
\end{gathered}
$$

The corresponding displacement and stress boundary conditions can be expressed as follows

$$
\begin{gathered}
\left\{\begin{array}{l}
w(0, y, t)=0, \frac{\partial^{2} w}{\partial x^{2}}(0, y, t)=0 \\
w(a, y, t)=0, \frac{\partial^{2} w}{\partial x^{2}}(a, y, t)=0
\end{array}\right. \\
\left\{\begin{array}{l}
w(x, 0, t)=0, \frac{\partial^{2} w}{\partial y^{2}}(x, 0, t)=0 \\
w(x, b, t)=0, \frac{\partial^{2} w}{\partial y^{2}}(x, b, t)=0
\end{array}\right. \\
\left\{\begin{array}{l}
\frac{\partial^{2} F}{\partial x^{2}}(0, y, t)=0 \\
\frac{\partial^{2} F}{\partial x^{2}}(a, y, t)=0
\end{array}\right. \\
\left\{\begin{array}{l}
\frac{\partial^{2} F}{\partial y^{2}}(x, 0, t)=0 \\
\frac{\partial^{2} F}{\partial y^{2}}(x, b, t)=0
\end{array}\right.
\end{gathered}
$$

\subsection{KBM perturbation method}

The functions satisfying the boundary condition Eq. (10) are separated as follows [45, 49]

$$
\begin{gathered}
w(x, y, t)=T_{m n}(t) \cdot W_{m n}(x, y) \\
F(x, y, t)=T_{m n}^{2}(t) \cdot F_{m n}(x, y)
\end{gathered}
$$

where $W_{m n}(x, y)$ is the given mode function, $T_{m n}(t)$ is a function of time, $m$ and $n$ are positive integers which represent the order of vibration mode in $\mathrm{x}$ and $\mathrm{y}$ directions respectively.

According to the basic vibration theory and boundary conditions, the displacement function can be obtained

$$
W(x, y)=\sin \frac{m \pi x}{a} \sin \frac{n \pi y}{b}
$$

Substitute Eq. (13) into Eq. (11)

$$
w(x, y, t)=T_{m n}(t) \sin \frac{m \pi x}{a} \sin \frac{n \pi y}{b}
$$

In order to simplify the computation symbols, we let $W_{m n}(x, y)=W(x, y)=W, T_{m n}(t)=T(t)=T, F_{m n}(x, y)=$ $F(x, y)=F$ and $F(x, y, t)=F$.

The substitution of Eq. (14) into Eq. (8) yields

$$
\begin{aligned}
\frac{1}{E_{1}} \frac{\partial^{4} F}{\partial y^{4}}+\frac{1}{E_{2}} \frac{\partial^{4} F}{\partial x^{4}} & =T^{2}(t) \frac{m^{2} n^{2} \pi^{4}}{2 a^{2} b^{2}}\left(\cos \frac{2 m \pi x}{a}\right. \\
& \left.+\cos \frac{2 n \pi y}{b}\right) \\
& +T(t) \sin \frac{m \pi x}{a} \sin \frac{n \pi y}{b} k_{0 x} \frac{n^{2} \pi^{2}}{b^{2}}
\end{aligned}
$$

The studied model is a symmetrical structure, so the stress function $F$ should be an even function and satisfy the stress boundary condition Eq. (10). We can suppose the solution of $F(x, y, t)$ is

$$
\begin{aligned}
F(x, y, t) & =T^{2}\left(\alpha \cos \frac{2 m \pi x}{a}+\beta \cos \frac{2 n \pi y}{b}+\delta_{1} x^{3}\right. \\
& \left.+\delta_{2} x^{2}+\delta_{3} x+\delta_{4} y^{3}+\delta_{5} y^{2}+\delta_{6} y+\delta_{7}\right) \\
& +T(t) \sin \frac{m \pi x}{a} \sin \frac{n \pi y}{b} \xi
\end{aligned}
$$

Assuming

$$
\left\{\begin{array}{l}
F(x, y, t)=T^{2}(t) \phi_{1}(x, y)+T(t) \phi_{2}(x, y) \\
\phi_{1}(x, y)=\alpha \cos \frac{2 m \pi x}{a}+\beta \cos \frac{2 n \pi y}{b}+\delta_{1} x^{3}+\delta_{2} x^{2}+\delta_{3} x \\
\quad+\delta_{4} y^{3}+\delta_{5} y^{2}+\delta_{6} y+\delta_{7} \\
\phi_{2}(x, y)=\xi \sin \frac{m \pi x}{a} \sin \frac{n \pi y}{b}=\xi W(x, y)
\end{array}\right.
$$

Substitution of Eq. (16) into Eq. (10) yields

$$
\begin{aligned}
\alpha & =\frac{E_{2} n^{2} a^{2}}{32 m^{2} b^{2}}, \quad \beta=\frac{E_{1} m^{2} b^{2}}{32 n^{2} a^{2}} \\
\xi & =\frac{k_{0 x}(n \pi / b)^{2}}{\left((n \pi / b)^{4} / E_{1}\right)+\left((m \pi / a)^{4} / E_{2}\right)} \\
\delta_{1} & =0, \quad \delta_{2}=\frac{\pi^{2} E_{2} n^{2}}{16 b^{2}} \quad \delta_{4}=0, \quad \delta_{5}=\frac{\pi^{2} E_{1} m^{2}}{16 a^{2}}
\end{aligned}
$$


According to the theory of differential equation, $\delta_{3}$, $\delta_{6}$ and $\delta_{7}$ are arbitrary constants. To simplify the calculation, set $\delta_{3}, \delta_{6}$ and $\delta_{7}$ to 0 . Substitution of Eq. (11) and Eq. (12) into Eq. (7) yields and converted into by the BubnovGalerkin method [50, 51].

$$
\iint_{s}\left\{\begin{array}{c}
\rho_{0} T^{\prime \prime}(t) W+c T^{\prime}(t) W-T(t) \\
\cdot\left(k_{0 x} h \frac{\partial^{2} \phi_{2}}{\partial y^{2}}+N_{0 x} \frac{\partial^{2} W}{\partial x^{2}}+N_{0 y} \frac{\partial^{2} W}{\partial y^{2}}\right)-T^{2}(t) \\
\cdot\left(k_{0 x} h \frac{\partial^{2} \phi_{1}}{\partial y^{2}}+h \frac{\partial^{2} \phi_{2}}{\partial y^{2}} \frac{\partial^{2} W}{\partial x^{2}}+h \frac{\partial^{2} \phi_{2}}{\partial x^{2}} \frac{\partial^{2} W}{\partial y^{2}}\right) \\
-T^{3}(t) \cdot\left(h \frac{\partial^{2} \phi_{1}}{\partial y^{2}} \frac{\partial^{2} W}{\partial x^{2}}+h \frac{\partial^{2} \phi_{1}}{\partial x^{2}} \frac{\partial^{2} W}{\partial y^{2}}\right)
\end{array}\right\}
$$

- $W(x, y) d x d y=0$

Eq. (17) can be transformed into a homogeneous differential equation

$$
\begin{aligned}
& A_{0} \cdot T^{\prime \prime}(t)+B_{0} \cdot T^{\prime}(t)+C_{0} \cdot T(t)+D_{0} \cdot T^{2}(t) \\
& +E_{0} \cdot T^{3}(t)=0
\end{aligned}
$$

where

$$
\begin{aligned}
A_{0} & =\iint_{s} \frac{\rho_{0}}{h} W^{2} d x d y=\frac{a b}{4} \rho_{0} \\
B_{0} & =\iint_{s} \frac{c}{h} W^{2} d x d y=\frac{a b}{4} c \\
C_{0} & =\iint_{S}-\left(k_{0 x} h \frac{\partial^{2} \phi_{2}}{\partial y^{2}}+N_{0 x} \frac{\partial^{2} W}{\partial x^{2}}+N_{0 y} \frac{\partial^{2} W}{\partial y^{2}}\right) W d x d y \\
& =\frac{m^{2} \pi^{2} b^{2} N_{0 x}+n^{2} \pi^{2} a^{2}\left(N_{0 y}+k_{0 x} h \xi\right)}{4 a b} \\
D_{0} & =\iint_{S}-\left(k_{0 x} h \frac{\partial^{2} \phi_{1}}{\partial y^{2}}+h \frac{\partial^{2} \phi_{2}}{\partial y^{2}} \frac{\partial^{2} W}{\partial x^{2}}\right. \\
& \left.+h \frac{\partial^{2} \phi_{2}}{\partial x^{2}} \frac{\partial^{2} W}{\partial y^{2}}\right) W d x d y \\
E_{0} & =\iint_{s}-\left(h \frac{\partial^{2} \phi_{1}}{\partial y^{2}} \frac{\partial^{2} W}{\partial x^{2}}+h \frac{\partial^{2} \phi_{1}}{\partial x^{2}} \frac{\partial^{2} W}{\partial y^{2}}\right) W d x d y \\
& =h \pi^{4} \frac{3 E_{1} m^{4} b^{4}+3 E_{2} n^{4} a^{4}}{64 a^{3} b^{3}}
\end{aligned}
$$

According to Eq. (18), it can be seen that the differential equation is an asymmetric nonlinear differential equation. Using KBM perturbation method [32] to solve Eq. (18), assuming that the disturbance parameter $\varepsilon=h^{2} / a b \ll 1$, Eq. (18) can be simplified from $x=x(t)=T(t)$ to

$$
\ddot{x}+\omega_{0}^{2} x=\varepsilon\left(\alpha_{1} x^{3}+\alpha_{2} x^{2}+\alpha_{3} \dot{x}\right)
$$

where

$$
\begin{aligned}
\omega_{0}^{2} & =\frac{C_{0}}{A_{0}}, \quad \alpha_{1}=-\frac{E_{0}}{\varepsilon A_{0}} \\
\alpha_{2} & =-\frac{D_{0}}{\varepsilon A_{0}}, \quad \alpha_{3}=-\frac{B_{0}}{\varepsilon A_{0}} \\
\ddot{x} & =\frac{d^{2} x}{d t^{2}}, \quad \dot{x}=\frac{d x}{d t}
\end{aligned}
$$

According to KBM perturbation method, $f(x, \ddot{x})=$ $\alpha_{1} x^{3}+\alpha_{2} x^{2}+\alpha_{3} \dot{x}$ and the solution of Eq. (19) can be obtained as follows

$$
\begin{gathered}
x=a \cos \chi \\
\frac{d x}{d t}=-a \omega_{0} \sin \chi
\end{gathered}
$$

where $\chi=\omega_{0} t+\theta, a$ and $\theta$ are constants determined by the initial conditions. In Eq. (20), $a$ and $\chi$ are defined as

$$
\begin{aligned}
& \frac{d a}{d t}=-\frac{\varepsilon}{\omega_{0}} A_{1}(a) \\
& \frac{d \chi}{d t}=\omega_{0}-\frac{\varepsilon}{a \omega_{0}} C_{1}(a)
\end{aligned}
$$

where

$$
\begin{aligned}
& A_{1}(a)=\frac{1}{2 \pi} \int_{0}^{2 \pi} \sin \chi f(x, \dot{x}) d \chi=-\frac{1}{2} \alpha_{3} a \omega_{0} \\
& C_{1}(a)=\frac{1}{2 \pi} \int_{0}^{2 \pi} \cos \chi f(x, \dot{x}) d \chi=\frac{3}{8} \alpha_{1} a^{3}
\end{aligned}
$$

Eq. (22) can be obtained by using the method of separating variables

$$
\begin{aligned}
& a=A e^{\frac{1}{2} \varepsilon \alpha_{3} t} \\
& \chi=\left(\omega_{0}-\frac{3 \varepsilon \alpha_{1} a^{2}}{8 \omega_{0}}\right) t+\chi_{0}
\end{aligned}
$$

In Eq. (23), $A$ represents the amplitude and $\chi_{0}$ represents the initial phase, which are determined by the initial conditions. Substitute Eq. (23) into Eq. (20), and get

$$
\begin{aligned}
x & =\chi(t) \\
& =A e^{\frac{1}{2} \varepsilon \alpha_{3} t} \cos \left(\left(\omega_{0}-\frac{3 \varepsilon \alpha_{1} A^{2} e^{\varepsilon \alpha_{3} t}}{8 \omega_{0}}\right) t+\chi_{0}\right)
\end{aligned}
$$

Eq. (24) is the approximate analytical solution of Eq. (19). According to Eq. (24), the approximate expression of frequency is

$$
\omega=\omega_{0}-\frac{3 \varepsilon \alpha_{1} A^{2} e^{\varepsilon \alpha_{3} t}}{8 \omega_{0}}
$$

Suppose the initial condition is

$$
\left\{\begin{array}{l}
\left.x(t)\right|_{t=0}=\frac{b_{0}}{\sin \frac{m \pi x_{0}}{a} \cdot \sin \frac{n \pi y_{0}}{b}} \\
\left.\frac{d x(t)}{d t}\right|_{t=0}=\frac{v_{0}}{\sin \frac{m \pi x_{0}}{a} \cdot \sin \frac{n \pi y_{0}}{b}}
\end{array}\right.
$$

where $b_{0}$ and $v_{0}$ are the initial displacement and initial velocity.

Substitute Eq. (23) into Eq. (21)

$$
\frac{d x(t)}{d t}=-A e^{\frac{1}{2} \alpha_{3} \varepsilon t}\left(\omega_{0}-\frac{3 \alpha_{1} \varepsilon A^{2} e^{\alpha_{3} \varepsilon t}}{8 \omega_{0}}\right)
$$




$$
\cdot \sin \left(\left(\omega_{0}-\frac{3 \alpha_{1} \varepsilon A^{2} e^{\alpha_{3} \varepsilon t}}{8 \omega_{0}}\right) t+\chi_{0}\right)
$$

Substitute the initial condition (26) into Eq. (20) and Eq. (21), and get

$$
\left\{\begin{array}{l}
\frac{b_{0}}{\sin \frac{m \pi x_{0}}{a} \cdot \sin \frac{n \pi y_{0}}{b}}=A \cos \chi_{0} \\
-A\left(\omega_{0}-\frac{3 \alpha_{1} \varepsilon A^{2}}{8 \omega_{0}}\right) \cdot \sin \chi_{0}=\frac{v_{0}}{\sin \frac{m \pi x_{0}}{a} \cdot \sin \frac{n \pi y_{0}}{b}}
\end{array}\right.
$$

In order to simplify the calculation, assume the initial displacement is $A$ and initial velocity is $v_{0}=0$. Substitute these conditions into Eq. (28) to get $\sin \chi_{0}=0$, and since $-\pi \leq \chi_{0} \leq \pi$, the initial phase is equal to 0 , the Eq. (24) can be simplified as

$$
x=T(t)=A e^{\frac{1}{2} \varepsilon \alpha_{3} t} \cos \left(\left(\omega_{0}-\frac{3 \varepsilon \alpha_{1} A^{2} e^{\varepsilon \alpha_{3} t}}{8 \omega_{0}}\right) t\right)
$$

By substituting Eq. (29) and Eq. (13) into Eq. (11), we can obtain the displacement function of damped nonlinear vibration of the skeleton supported prestressed membrane structure.

$$
\begin{aligned}
w(x, y, t) & =\sum_{m=1}^{\infty} \sum_{n=1}^{\infty} \sin \frac{m \pi x}{a} \sin \frac{n \pi y}{b} \\
& \cdot\left(A e^{\frac{1}{2} \alpha_{3} \varepsilon t} \cdot \cos \left(\left(\omega_{0}-\frac{3 \alpha_{1} \varepsilon A^{2} e^{\alpha_{3} \varepsilon t}}{8 \omega_{0}}\right) t\right)\right)
\end{aligned}
$$

According to Eq. (30), the transverse displacement of any point on the membrane can be obtained. At the same time, the displacement time-history curve of the membrane can also be analyzed according to Eq. (30).

By superposing the initial surface Eq. (3) of the skeleton supported membrane with its displacement Eq. (30), the modes $S$ of the skeleton supported membrane can be obtained

$$
\begin{gathered}
S=-\frac{\kappa_{2}(x-(a / 2))^{2}}{(a / 2)^{2}}+\sum_{m=1}^{\infty} \sum_{n=1}^{\infty} \sin \frac{m \pi x}{a} \sin \frac{n \pi y}{b} \\
\cdot\left(A e^{\frac{1}{2} \alpha_{3} \varepsilon t} \cdot \cos \left(\left(\omega_{0}-\frac{3 \alpha_{1} \varepsilon A^{2} e^{\alpha_{3} \varepsilon t}}{8 \omega_{0}}\right) t\right)\right)
\end{gathered}
$$

where

$$
\begin{aligned}
\omega_{0} & =\sqrt{\frac{m^{2} \pi^{2} b^{2} N_{0 x}+n^{2} \pi^{2} a^{2}\left(N_{0 y}+k_{0 x} h \xi\right)}{a^{2} b^{2} \rho_{0}}} \\
\alpha_{1} & =-\pi^{4} \frac{3 E_{1} m^{4} b^{4}+3 E_{2} n^{4} a^{4}}{16 a^{3} b^{3} h \rho_{0}} \alpha_{3}=-\frac{a b c}{h^{2} \rho_{0}} \\
\xi & =\frac{k_{0 x}(n \pi / b)^{2}}{\left((n \pi / b)^{4} / E_{1}\right)+\left((m \pi / a)^{4} / E_{2}\right)} \\
k_{0 x} & =-\frac{8 \kappa_{2}}{a^{2}} \varepsilon=\frac{h^{2}}{a b}
\end{aligned}
$$

\section{Runge-Kutta method verification}

Runge-Kutta method is a high-precision one-step algorithm widely used in engineering to solve nonlinear differential equation. Because of the high accuracy of this algorithm, the fourth order Runge-Kutta method was used to solve the displacement numerical solution. The effective range of the KBM perturbation solution can be estimated by comparing the KBM perturbation solution with the Runge-Kutta method.

We take the anisotropy membrane materials commonly used in engineering practice as an example. Young's modulus in the $\mathrm{x}$ and $\mathrm{y}$ directions are $E_{1}=1.4 \times 10^{6} \mathrm{kN} / \mathrm{m}^{2}$ and $E_{2}=0.9 \times 10^{6} \mathrm{kN} / \mathrm{m}^{2}$ [52-54]. The density of the membrane is $\rho_{0}=1.7 \mathrm{~kg} / \mathrm{m}^{2}$. Viscous damping is $c=120 \mathrm{Ns} / \mathrm{m}$. The thickness of the membrane is $h=1.0 \mathrm{~mm}$. The length of the membrane is $a=1 \mathrm{~m}$ and the width is $b=1 \mathrm{~m}$. The prestress is $N_{0 x}=N_{0 y}=1 \mathrm{kN} / \mathrm{m}$.

Figures 2 and 3 show the results of the first order analytical and numerical solutions for different initial displacements when the rise span ratio is $\kappa_{2}=1 / 10$.

It can be seen from Figures 2-3 that when the initial displacement $A \in[0,0.06] \mathrm{m}$, the error of the maximum

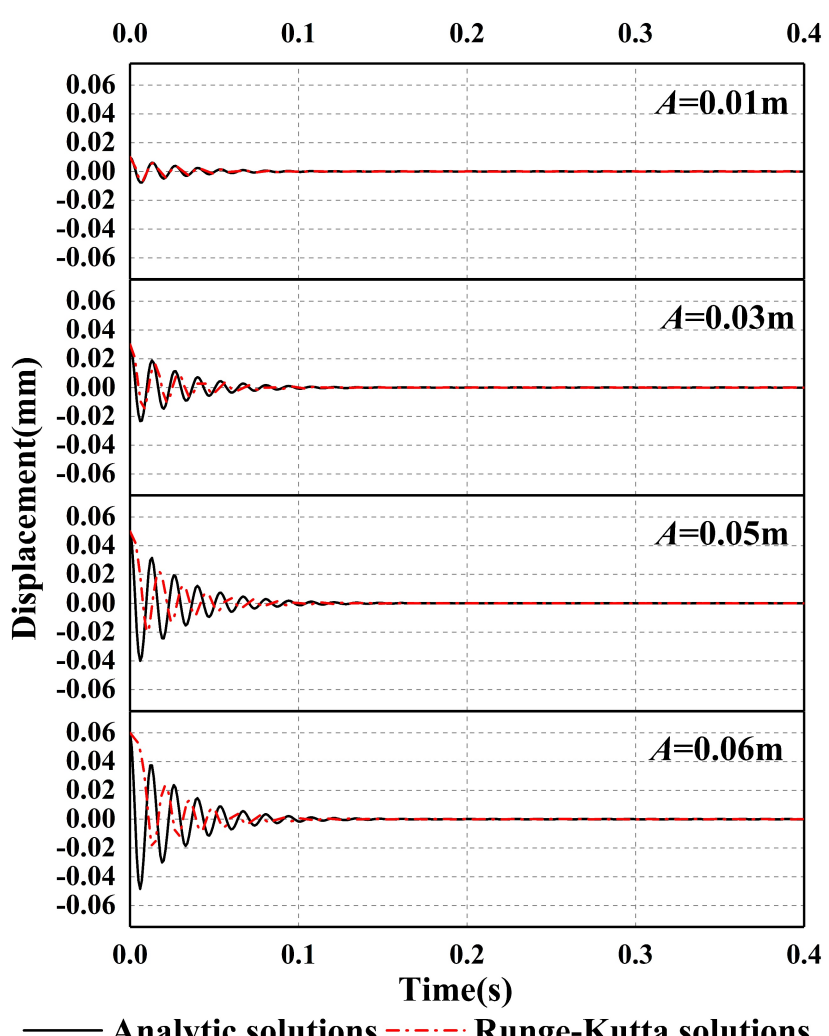

Figure 2: Comparison of analytical and numerical solutions of $A \in[0,0.06] \mathrm{m}$ 


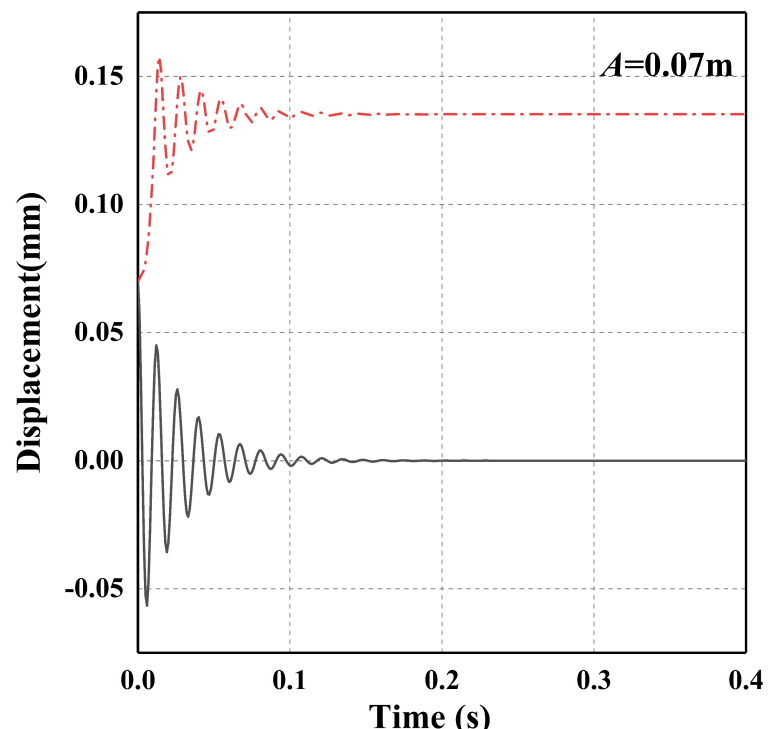

Analytic solutions ----- Runge-Kutta solutions

Figure 3: Comparison of analytical solution and numerical solution when $A>0.06 \mathrm{~m}$ displacement is less than $30 \%$, and the analytical solution and numerical solution are relatively close. However, the error of the maximum displacement is $49.6 \%$ when $A>$ $0.06 \mathrm{~m}$. The analytical solution is greatly different from the numerical solution. Thus, it can be judged that the analytical solution obtained by KBM perturbation method is valid in the range of initial displacement $A \in[0,0.06] \mathrm{m}$. When the initial displacement is greater than $0.06 \mathrm{~m}$, it is not accurate to use KBM to solve the nonlinear differential equation of cylindrical membrane.

\section{Analytical and numerical examples}

\subsection{Analytical calculation}

The same parameters as above were used for example calculation and analysis.

Table 1: The frequency (rad/s) under different initialdisplacements and time instants when the rise span ratio is $1 / 10$

\begin{tabular}{|c|c|c|c|c|c|c|}
\hline \multicolumn{7}{|c|}{ The initial displacements $A$} \\
\hline Order & $t(\mathrm{~s})$ & 0.01 & 0.02 & 0.04 & 0.06 & $A \rightarrow 0$ \\
\hline \multirow[t]{8}{*}{ 1st order } & 0.000 & 468.73 & 474.69 & 498.51 & 538.22 & 466.75 \\
\hline & 0.005 & 468.14 & 472.33 & 489.07 & 516.96 & \\
\hline & 0.010 & 467.73 & 470.67 & 482.43 & 502.03 & \\
\hline & 0.020 & 467.23 & 468.68 & 474.49 & 484.16 & \\
\hline & 0.040 & 466.86 & 467.22 & 468.63 & 470.99 & \\
\hline & 0.080 & 466.75 & 466.77 & 466.86 & 467.00 & \\
\hline & 0.100 & 466.75 & 466.75 & 466.77 & 466.81 & \\
\hline & $t \rightarrow \infty$ & \multicolumn{4}{|c|}{466.75} & \\
\hline \multirow[t]{8}{*}{ 2nd order } & 0.000 & 532.17 & 620.66 & 856.42 & 1034.80 & 502.68 \\
\hline & 0.005 & 523.40 & 585.57 & 813.98 & 939.31 & \\
\hline & 0.010 & 517.24 & 560.92 & 784.16 & 872.22 & \\
\hline & 0.020 & 509.87 & 531.40 & 617.69 & 761.45 & \\
\hline & 0.040 & 504.43 & 509.69 & 530.71 & 565.75 & \\
\hline & 0.080 & 502.78 & 503.10 & 504.34 & 506.42 & \\
\hline & 0.100 & 502.70 & 502.78 & 503.08 & 503.59 & \\
\hline & $t \rightarrow \infty$ & \multicolumn{4}{|c|}{502.68} & \\
\hline \multirow[t]{8}{*}{3 rd order } & 0.000 & 722.63 & 749.39 & 974.59 & 1564.48 & 713.71 \\
\hline & 0.005 & 719.98 & 738.78 & 834.25 & 1248.72 & \\
\hline & 0.010 & 718.12 & 731.33 & 735.65 & 1026.86 & \\
\hline & 0.020 & 715.89 & 722.41 & 748.49 & 791.97 & \\
\hline & 0.040 & 714.24 & 715.83 & 722.19 & 732.78 & \\
\hline & 0.080 & 713.74 & 713.84 & 714.22 & 714.85 & \\
\hline & 0.100 & 713.72 & 713.74 & 713.84 & 713.99 & \\
\hline & $t \rightarrow \infty$ & \multicolumn{4}{|c|}{713.71} & \\
\hline
\end{tabular}




\subsubsection{Frequency calculation}

It can be seen from Eq. (25) that the frequencies of each order vary with the initial displacement and time. Under different initial displacement and time, the first three frequencies of membrane material with rise span ratio $\kappa_{2}=1 / 10$ are respectively calculated according to Eq. (25). The calculation results of the first three order nonlinear vibration frequency with different initial displacements and rise span ratio $\kappa_{2}=1 / 10$ are shown in Table 1 .

Analysis of Table 1 shows that:

(1) When considering the damping and geometric nonlinearity of membrane vibration, the nonlinear vibration frequency of membrane increases with the increase of initial displacement and order at a certain time.

(2) For the vibration frequency of each order, the natural frequency value of the nonlinear vibration of each order can be obtained when the initial displacement $A \rightarrow 0$ or time $t \rightarrow \infty$.

(3) The natural frequency of the nonlinear vibration of the membrane increases with the increase of order.

\subsubsection{Vibration mode}

By substituting the geometry and material parameters of the membrane into Eq. (30) and Eq. (31), the vibration mode of the first four orders can be obtained. It is assumed that the initial displacement of nonlinear vibration is $A=0.05 \mathrm{~m}$. According to Eq. (31), the modes of the first four orders are plotted at $\mathrm{t}=0 \mathrm{~s}$ and $t=0.0035 \mathrm{~s}$, as shown in Figure 4. In Figure 4, the coordinate unit is meters (m).

From the results of modal analysis, it can be seen that Eq. (31) can be used to calculate the vibration modal equations of each order conveniently, and the superposition vibration modes of nonlinear free vibration of viscous damping skeleton supported membrane can also be obtained. In the actual structure, the influence of low order mode is greater than that of high order mode. Therefore, only the first four modes are analyzed.

\subsubsection{The displacement time history}

By using Eq. (30), the time-history curve functions of nonlinear vibration displacements at characteristic points on the membrane are calculated respectively. The first four orders time-history curves of displacement are shown in Figure 6. Rise span ratio $\kappa_{2}=1 / 10$. Coordinates of these feature points are A $(x=0.5 \mathrm{~m}, y=0.5 \mathrm{~m}), \mathrm{B}(x=0.75 \mathrm{~m}, y$ $=0.25 \mathrm{~m}), \mathrm{C}(x=0.75 \mathrm{~m}, y=0.75 \mathrm{~m}), \mathrm{D}(x=0.25 \mathrm{~m}, y=0.25$ $\mathrm{m})$ and $\mathrm{E}(x=0.25 \mathrm{~m}, y=0.75 \mathrm{~m})$. These feature points are shown in Figure 5, and the dimension of ordinate is meter.

The analysis of Figure 6 shows that:

(1) The displacement time history curves of each feature point on the membrane are obtained by superimposing the first four orders of vibration. Because of the damped vibration, the displacement of each feature point decreases with the increase of time, and the membrane vibration will stop when the final displacement approaches zero.

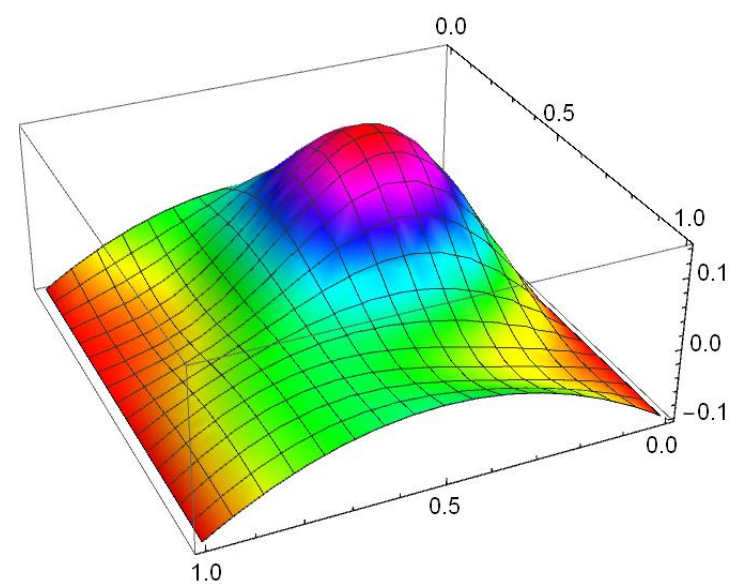

(a) $\mathrm{t}=0 \mathrm{~s}$

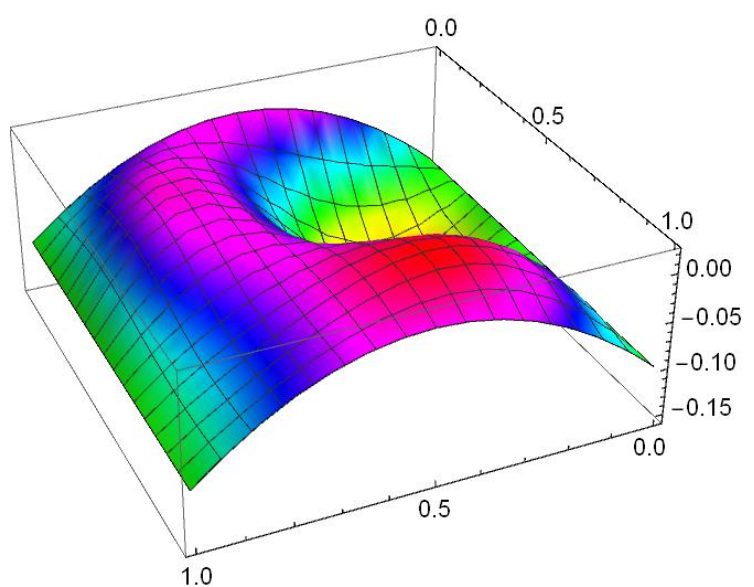

(b) $\mathrm{t}=0.0035 \mathrm{~s}$

Figure 4: Superposition of the first four modes 


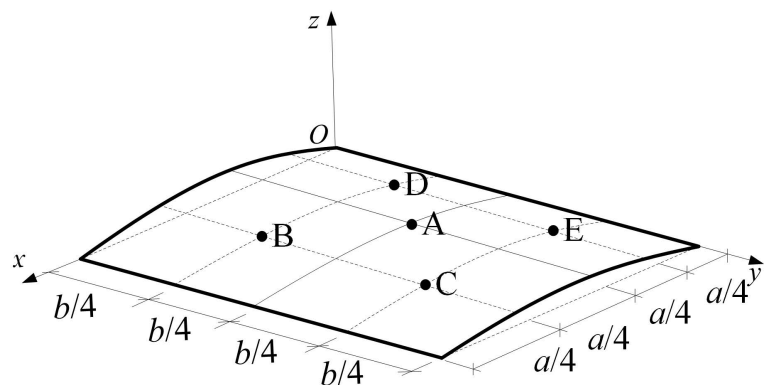

Figure 5: Feature points on membrane surface

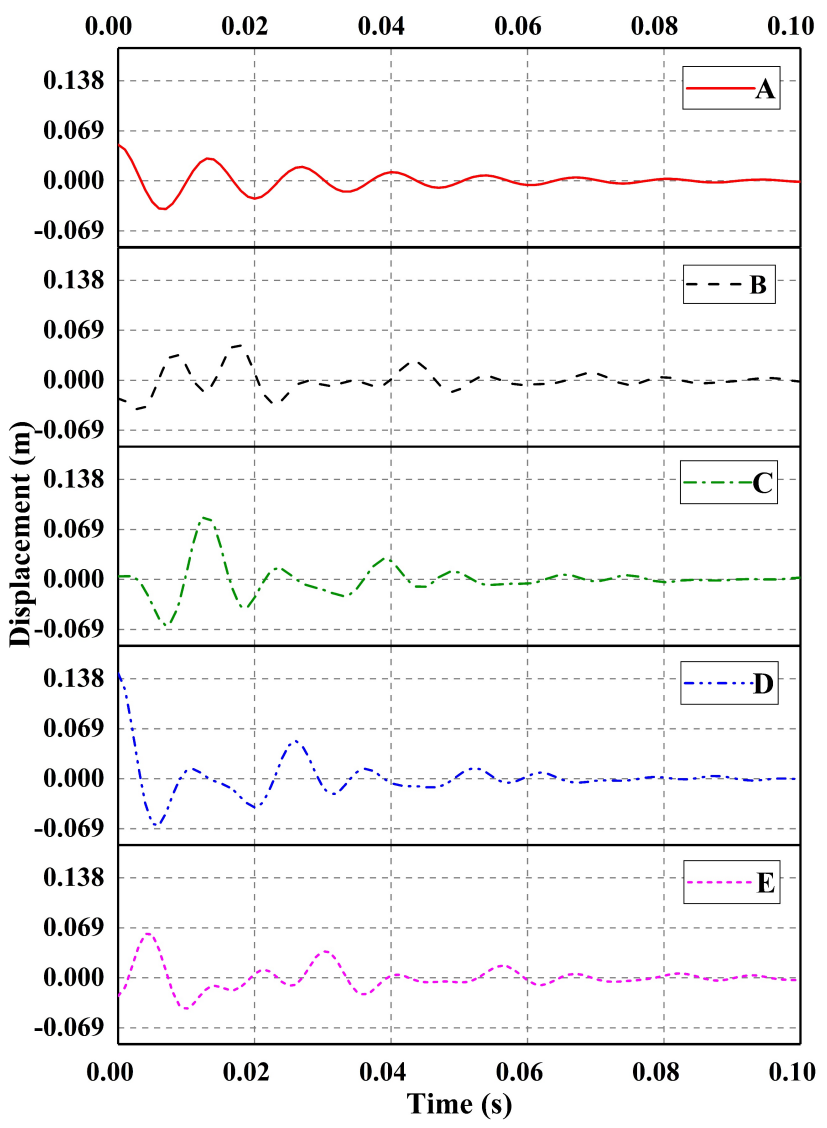

Figure 6: Displacement time-history curves of each feature point of nonlinear vibration
(2) Because point A is located in the center of the membrane, and the distances from point A to each boundary of the membrane are the same. After superposition, all the higher harmonics of point $A$ are canceled each other. Therefore, the vibration displacement of point $\mathrm{A}$ is regular.

\subsection{Comparison and analysis}

Mathematica was used to calculate the analytical solution of KBM perturbation method, and the ode45 function in Matlab was used to realize the numerical solution of fourth order Runge-Kutta method. The influence of different prestress, elastic modulus ratio, length width ratio, viscous damping and material density on the nonlinear vibration of the center point $\mathrm{A}$ of the membrane were analyzed, and the results of analytical solution and numerical solution were compared and analyzed.

\subsubsection{Effect of prestress}

In order to analyze the influence of prestress on the natural frequency of the membrane, the first three natural frequencies of nonlinear vibration with different prestressing forces under the rise span ratio $\kappa_{2}=1 / 10$ and $\kappa_{2}=1 / 12$ are calculated according to Eq. (25). Assuming that initial displacement of nonlinear vibration is $A=0.05 \mathrm{~m}$, Young's modulus in the $x$ and $y$ directions are $E_{1}=1.4 \times 10^{6} \mathrm{kN} / \mathrm{m}^{2}$ and $E_{2}=0.9 \times 10^{6} \mathrm{kN} / \mathrm{m}^{2}$ [42]. The density of the membrane is $\rho_{0}=1.7 \mathrm{~kg} / \mathrm{m}^{2}$. Viscous damping is $c=120 \mathrm{Ns} / \mathrm{m}$. The thickness of the membrane is $h=1.0 \mathrm{~mm}$. The length and width of the membrane are $a=1 \mathrm{~m}$ and $b=1 \mathrm{~m}$. The calculation results are shown in Tables 2-3 and Figures 7-8.

By analyzing Tables $2-3$ and Figures $7-8$, the following conclusions can be drawn:

(1) The frequencies of the membrane nonlinear vibration increases with the increase of prestress, and the

Table 2: The natural frequencies ( $\mathrm{rad} / \mathrm{s})$ of analytical solutions under different prestress and rise span ratios

\begin{tabular}{cccccccc}
\hline$\kappa_{2}$ & $N_{0 x}=N_{0 y}(\mathrm{kN} / \mathrm{m})$ & 1 & 10 & 20 & 30 & 40 & 50 \\
\hline $1 / 10$ & 1st order & 466.75 & 567.76 & 662.17 & 744.70 & 818.96 & 887.02 \\
& 2nd order & 502.68 & 818.96 & 1030.01 & 1162.41 & 1281.21 & 1389.88 \\
& 3rd order & 713.71 & 877.86 & 1065.43 & 1264.75 & 1436.68 & 1590.13 \\
$1 / 12$ & 1st order & 393.49 & 509.25 & 612.74 & 701.11 & 779.54 & 850.76 \\
& 2nd order & 435.51 & 779.54 & 956.11 & 1097.46 & 1222.58 & 1336.04 \\
& 3rd order & 602.17 & 789.85 & 1035.44 & 1239.59 & 1414.58 & 1570.19 \\
\hline
\end{tabular}


Table 3: The natural frequencies (rad/s) of numerical solutions under different prestress and rise span ratios

\begin{tabular}{cccccccc}
\hline$\kappa_{2}$ & $N_{0 x}=N_{0 y}(\mathrm{kN} / \mathrm{m})$ & 1 & 10 & 20 & 30 & 40 & 50 \\
\hline $1 / 10$ & 1st order & 447.68 & 557.63 & 659.73 & 738.27 & 816.81 & 887.81 \\
& 2nd order & 746.44 & 919.23 & 1060.60 & 1186.27 & 1303.76 & 1413.72 \\
& 3rd order & 769.69 & 950.65 & 1146.68 & 1327.64 & 1484.72 & 1626.09 \\
$1 / 12$ & 1st order & 361.28 & 502.65 & 612.61 & 699.32 & 777.86 & 848.23 \\
& 2nd order & 683.61 & 840.69 & 989.60 & 1123.43 & 1249.10 & 1359.05 \\
& 3rd order & 706.86 & 919.23 & 1123.43 & 1303.76 & 1460.84 & 1610.38 \\
\hline
\end{tabular}

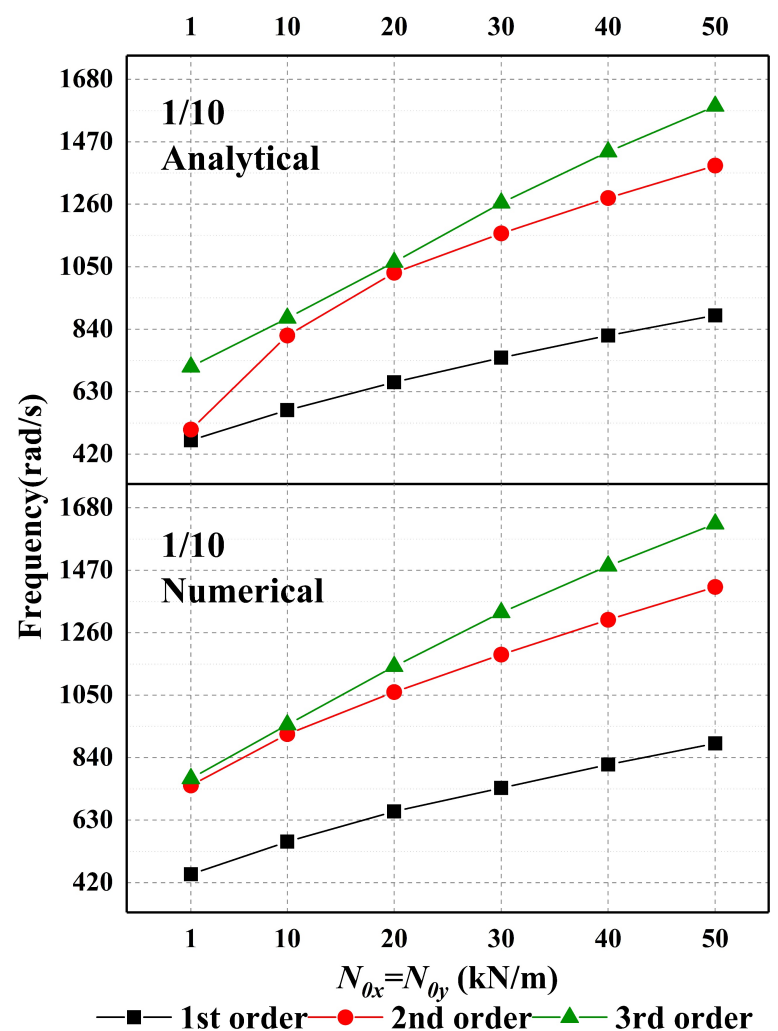

Figure 7: Comparison of natural frequencies under different prestressing conditions when the rise span ratio is $1 / 10$

frequency increases with the increase of order under the same prestress.

(2) Under the same conditions, the frequency value of every order with rise span ratio $\kappa_{2}=1 / 12$ is smaller than that with rise span ratio $\kappa_{2}=1 / 10$.

(3) The frequency of numerical solution is generally higher than that of analytical solution, but the general trend is basically the same.

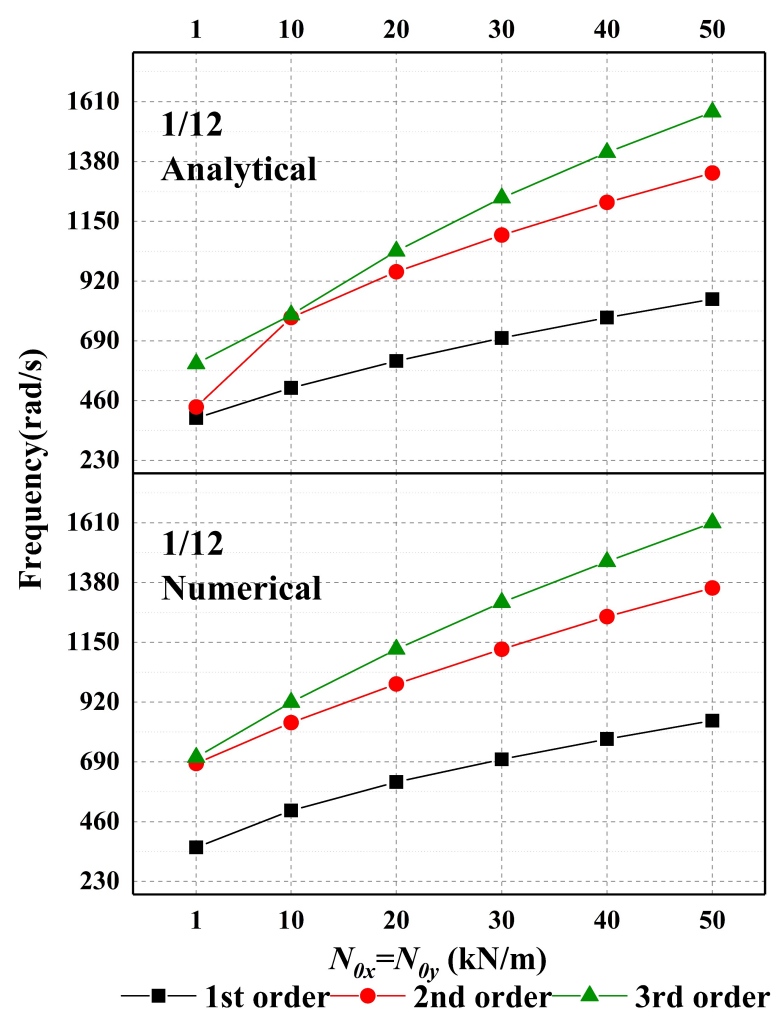

Figure 8: Comparison of natural frequencies under different prestressing conditions when the rise span ratio is $1 / 12$

\subsubsection{Effect of elastic modulus ratio}

In order to consider the influence of the elastic modulus ratio $\left(E_{1}, E_{2}\right)$ on nonlinear vibration, the first three natural frequencies of nonlinear vibration with different elastic modulus ratios under the rise span ratios $\kappa_{2}=1 / 10$ and $\kappa_{2}=1 / 12$ are calculated according to Eq. (25). Similarly, assuming that initial displacement of nonlinear vibration is $A=0.05 \mathrm{~m}$ and other material parameters invariant. The results are shown in Tables 4-5 and Figures 9-10.

From Tables 4-5 and Figures 9-10, we can conclude that:

(1) When $E_{1}>E_{2}$ and value of $E_{2}$ is fixed, the natural frequencies of nonlinear vibration decreases as 
Table 4: The natural frequencies ( $\mathrm{rad} / \mathrm{s})$ of analytical solutions under different elastic modulus ratios and rise span ratios

\begin{tabular}{ccccccccccc}
\hline$\kappa_{2}$ & $\begin{array}{c}E_{1}, E_{2} \\
\left(\times 10^{6} \mathrm{kN} / \mathrm{m}^{2}\right)\end{array}$ & 5,1 & 4,1 & 3,1 & 2,1 & 1,1 & 1,2 & 1,3 & 1,4 & 1,5 \\
\hline $1 / 10$ & 1st order & 570.38 & 559.27 & 542.19 & 512.44 & 447.04 & 512.44 & 542.19 & 559.27 & 570.38 \\
& 2nd order & 600.14 & 589.59 & 573.41 & 545.37 & 484.44 & 545.37 & 573.41 & 589.59 & 600.14 \\
& 3rd order & 1209.63 & 1110.74 & 990.01 & 835.65 & 619.16 & 627.77 & 630.73 & 632.22 & 633.13 \\
$1 / 12$ & 1st order & 479.04 & 469.85 & 455.73 & 431.17 & 377.27 & 431.17 & 455.73 & 469.85 & 479.04 \\
& 2nd order & 514.11 & 505.57 & 492.47 & 469.83 & 420.91 & 469.83 & 492.47 & 505.57 & 514.11 \\
& 3rd order & 1012.41 & 930.39 & 830.36 & 702.71 & 524.49 & 531.55 & 533.98 & 535.21 & 535.95 \\
\hline
\end{tabular}

Table 5: The natural frequencies (rad/s) of numerical solutions under different elastic modulus ratios and rise span ratios

\begin{tabular}{ccccccccccc}
\hline$\kappa_{2}$ & $\begin{array}{c}E_{1}, E_{2} \\
\left(\times 10^{6} \mathrm{kN} / \mathrm{m}^{2}\right)\end{array}$ & 5,1 & 4,1 & 3,1 & 2,1 & 1,1 & 1,2 & 1,3 & 1,4 & 1,5 \\
\hline $1 / 10$ & 1st order & 549.78 & 541.92 & 526.22 & 494.80 & 424.12 & 471.24 & 557.63 & 565.49 & 596.90 \\
& 2nd order & 715.03 & 644.03 & 636.49 & 596.90 & 510.51 & 620.46 & 715.03 & 801.11 & 824.98 \\
& 3rd order & 1374.76 & 1249.10 & 1099.56 & 919.23 & 675.44 & 722.57 & 769.69 & 809.27 & 856.40 \\
$1 / 12$ & 1st order & 463.38 & 447.68 & 431.97 & 400.55 & 298.45 & 408.41 & 526.22 & 541.92 & 573.34 \\
& 2nd order & 549.78 & 518.36 & 515.22 & 479.09 & 463.38 & 486.95 & 534.07 & 596.90 & 659.73 \\
& 3rd order & 1036.73 & 942.48 & 840.69 & 809.27 & 596.90 & 652.19 & 699.32 & 753.98 & 793.57 \\
\hline
\end{tabular}

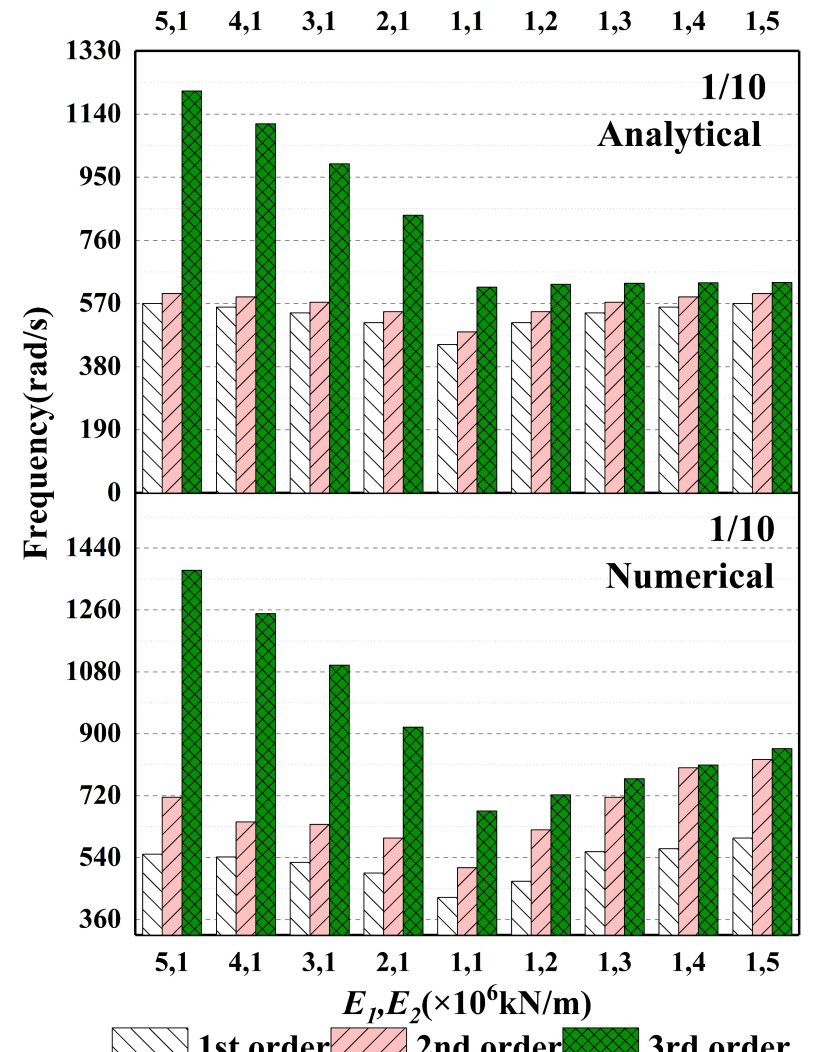

Figure 9: Comparison of natural frequencies under different elastic modulus ratios when the rise span ratio is $1 / 10$

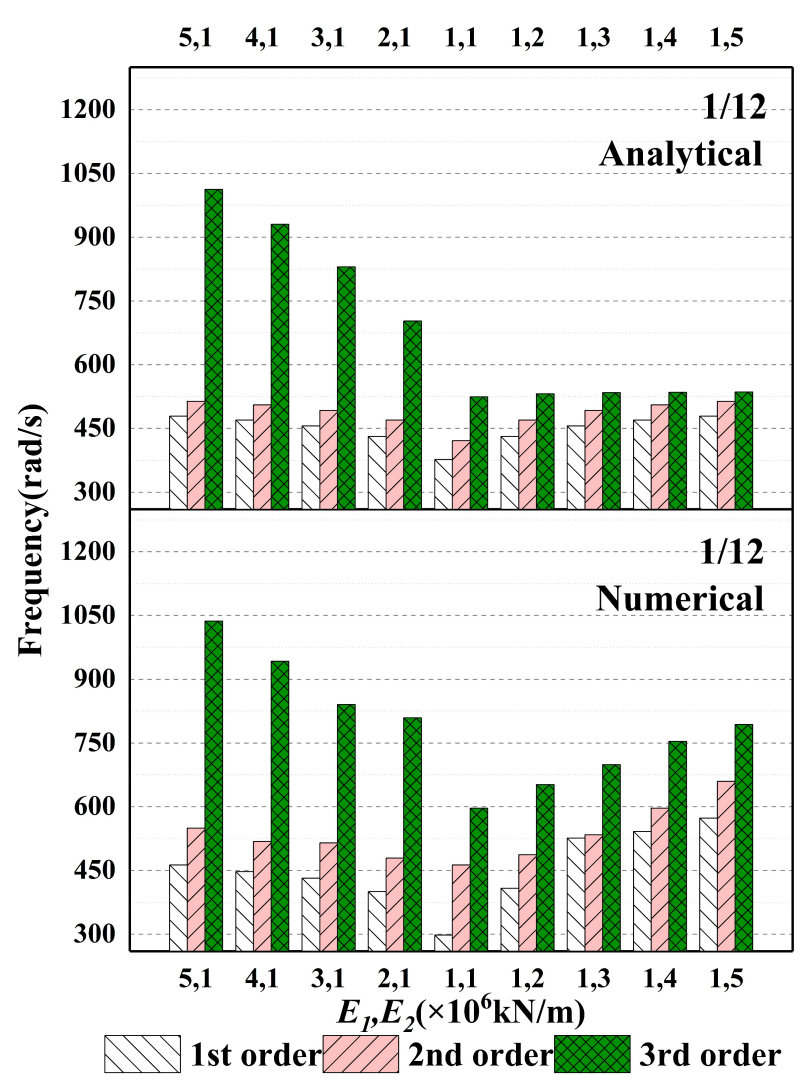

Figure 10: Comparison of natural frequencies under different elastic modulus ratios when the rise span ratio is $1 / 12$ 
the ratio of elastic modulus decreases, and increases with the order increases. When $E_{1}<E_{2}$ and value of $E_{1}$ is fixed, the frequency of nonlinear vibration increases as the ratio of elastic modulus decreases, and increases with the order increases.

(2) All other conditions being equal, the natural frequency of nonlinear vibration changes when exchanging the values of $E_{1}$ and $E_{2}$.

(3) The natural frequency values of nonlinear vibration are the smallest when the ratio of elastic modulus is 1:1, and the frequencies at the rise span ratio of $\kappa_{2}=1 / 12$ are less than the frequencies at the rise span ratio of $\kappa_{2}=1 / 10$.

(4) In conclusion, the ratio of elastic modulus and the rise span ratio have a great influence on the natural vibration frequency, and the result of the theoretical analytical solution is smaller than that of the numerical solution.

\subsubsection{Effect of length width ratio}

In order to consider the influence of the length width ratio $(a, b)$ of the membrane on nonlinear vibration, the first

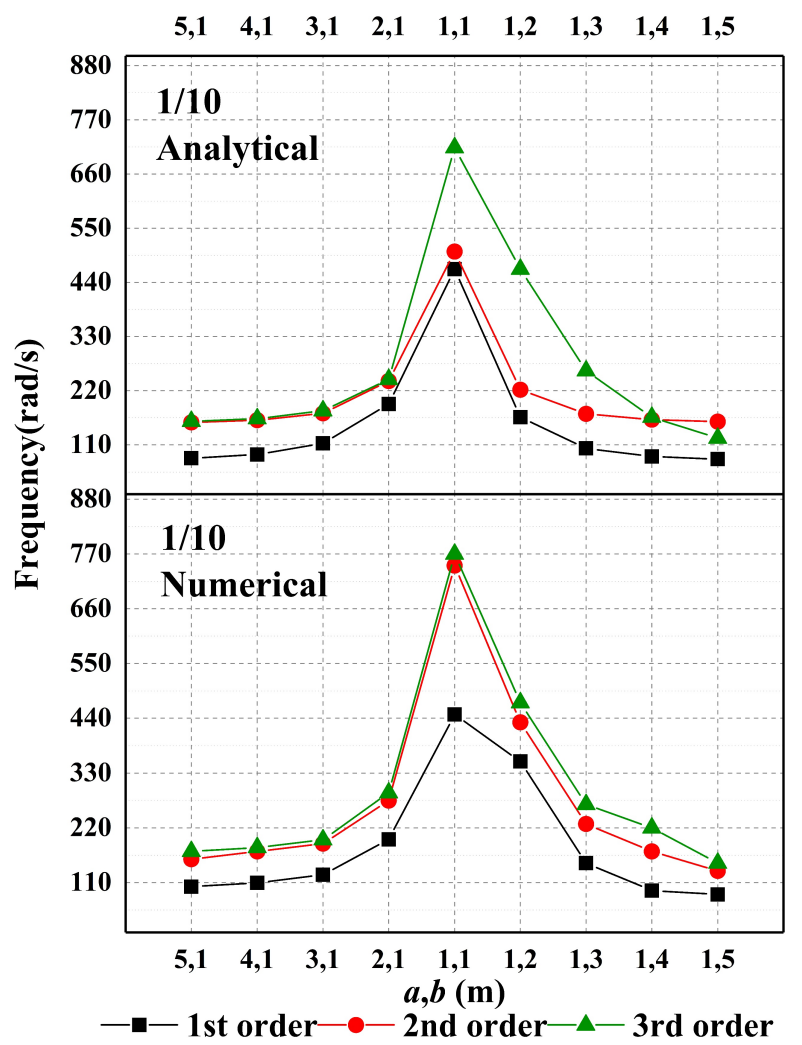

Figure 11: Comparison of natural frequencies under different length width ratios when the rise span ratio is $1 / 10$ three order natural frequencies of nonlinear vibration with different length width ratios under the rise span ratios and $\kappa_{2}=1 / 12$ are respectively calculated according to Eq. (25). Assuming that initial displacement of nonlinear vibration is $A=0.05 \mathrm{~m}$ and other material parameters remain unchanged. The results are shown in Tables 6-7 and Figures 11-12.

From Tables 6-7 and Figures 11-12, we can conclude that:

(1) If the length width ratios of two orthogonal directions are swapped, the natural frequency values of nonlinear vibration will be different compared to that before the exchange.

(2) When the rise span ratio $\kappa_{2}$ of membrane structure changes from $1 / 10$ to $1 / 12$, the natural frequency values of nonlinear vibration will decrease accordingly.

(3) The natural frequencies of the numerical solution are greater than those of the analytical solution.

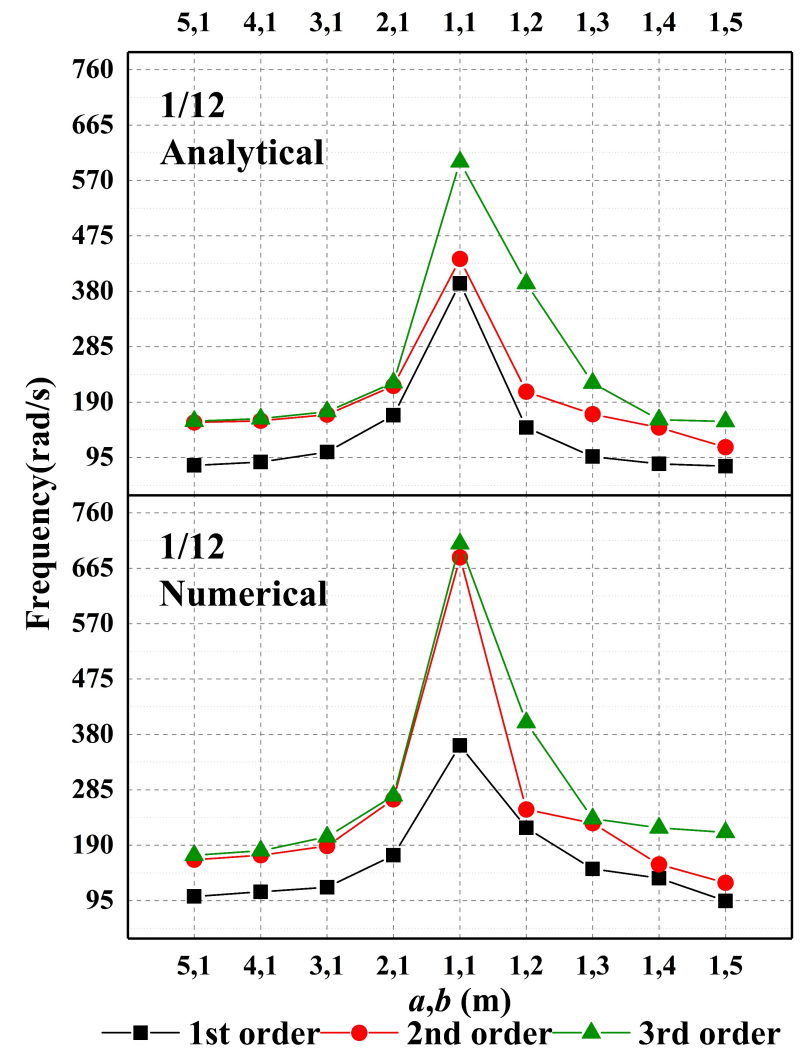

Figure 12: Comparison of natural frequencies under different length width ratios when the rise span ratio is $1 / 12$ 
Table 6: The natural frequencies ( $\mathrm{rad} / \mathrm{s}$ ) of analytical solutions under different length width ratios and rise span ratios

\begin{tabular}{ccccccccccc}
\hline$\kappa_{2}$ & $a, b(\mathrm{~m})$ & 5,1 & 4,1 & 3,1 & 2,1 & 1,1 & 1,2 & 1,3 & 1,4 & 1,5 \\
\hline $1 / 10$ & 1st order & 82.94 & 90.64 & 113.29 & 193.08 & 466.75 & 166.18 & 102.96 & 86.54 & 81.11 \\
& 2nd order & 155.88 & 160.14 & 174.26 & 239.62 & 502.68 & 222.23 & 173.07 & 161.23 & 157.14 \\
& 3rd order & 158.09 & 163.46 & 179.41 & 243.00 & 713.71 & 466.75 & 260.33 & 166.18 & 123.56 \\
$1 / 12$ & 1st order & 81.38 & 87.12 & 104.33 & 167.65 & 393.49 & 146.27 & 96.61 & 84.17 & 80.09 \\
& 2nd order & 155.05 & 158.16 & 168.47 & 217.74 & 435.51 & 207.77 & 169.37 & 146.27 & 112.52 \\
& 3rd order & 157.28 & 161.54 & 173.89 & 223.33 & 602.17 & 393.49 & 222.77 & 159.97 & 156.61 \\
\hline
\end{tabular}

Table 7: The natural frequencies ( $\mathrm{rad} / \mathrm{s}$ ) of numerical solutions under different length width ratios and rise span ratios

\begin{tabular}{ccccccccccc}
\hline$\kappa_{2}$ & $a, b(\mathrm{~m})$ & 5,1 & 4,1 & 3,1 & 2,1 & 1,1 & 1,2 & 1,3 & 1,4 & 1,5 \\
\hline $1 / 10$ & 1st order & 102.10 & 109.96 & 125.66 & 196.98 & $\mathbf{4 4 7 . 6 8}$ & 353.43 & 149.23 & 94.25 & 86.39 \\
& 2nd order & 157.08 & 172.79 & 188.50 & 274.89 & $\mathbf{7 4 6 . 4 4}$ & 431.97 & 227.77 & 172.79 & 133.52 \\
& 3rd order & 172.79 & 180.64 & 196.35 & 290.60 & $\mathbf{7 6 9 . 6 9}$ & 471.24 & 267.04 & 219.91 & 149.23 \\
$1 / 12$ & 1st order & 102.10 & 109.96 & 117.81 & 172.79 & $\mathbf{3 6 1 . 2 8}$ & 219.91 & 149.23 & 133.52 & 94.25 \\
& 2nd order & 154.93 & 172.79 & 188.50 & 268.61 & $\mathbf{6 8 3 . 6 1}$ & 251.33 & 227.77 & 157.08 & 125.66 \\
& 3rd order & 172.79 & 180.64 & 204.20 & 274.89 & $\mathbf{7 0 6 . 8 6}$ & 400.55 & 235.62 & 219.91 & 212.06 \\
\hline
\end{tabular}

\subsubsection{Effect of viscous damping}

In order to analyze the effect of viscous damping on nonlinear vibration, the first nonlinear vibration displacements of different viscous damping at rise span ratio $\kappa_{2}=1 / 10$ are calculated respectively. Assuming that the initial displacement of nonlinear vibration is $A=0.05 \mathrm{~m}$ and other material parameters invariant, the calculation results are shown in Figure 13.
By analyzing Figure 13, it can be known that:

(1) The displacement of membrane nonlinear vibration gradually decreases to 0 with the increase of time, and presents periodic changes.

(2) With the increase of viscous damping, the displacement attenuation speed of nonlinear vibration of membrane is accelerated.

Table 8: The natural frequencies (rad/s) of analytical solutions under different material densities and rise span ratios

\begin{tabular}{lccccccc}
\hline & $\rho_{0} \mathrm{~kg} / \mathrm{m}^{2}$ & 0.7 & 1.2 & 1.7 & 2.2 & 2.7 & 3.2 \\
\hline $1 / 10$ & 1st order & 741.30 & 577.27 & $\mathbf{4 6 6 . 7 5}$ & $\mathbf{4 1 0 . 2 9}$ & $\mathbf{3 7 0 . 3 6}$ & $\mathbf{3 4 0 . 2 0}$ \\
& 2nd order & 990.31 & 921.17 & $\mathbf{7 0 2 . 6 8}$ & $\mathbf{4 4 1 . 8 8}$ & $\mathbf{3 9 8 . 8 7}$ & $\mathbf{3 6 6 . 3 9}$ \\
& 3rd order & 1194.62 & 978.02 & $\mathbf{7 1 3 . 7 1}$ & $\mathbf{6 2 7 . 3 9}$ & $\mathbf{5 6 6 . 3 3}$ & $\mathbf{5 2 0 . 2 0}$ \\
$1 / 12$ & 1st order & 629.73 & 494.12 & $\mathbf{3 9 3 . 4 9}$ & $\mathbf{3 4 5 . 9 0}$ & $\mathbf{3 1 2 . 2 3}$ & $\mathbf{2 8 6 . 8 0}$ \\
& 2nd order & 917.55 & 869.06 & 435.51 & $\mathbf{3 8 2 . 8 3}$ & $\mathbf{3 4 5 . 5 7}$ & $\mathbf{3 1 7 . 4 3}$ \\
& 3rd order & 1036.06 & 891.02 & $\mathbf{6 0 2 . 1 7}$ & $\mathbf{5 2 9 . 3 4}$ & $\mathbf{4 7 7 . 8 2}$ & $\mathbf{4 3 8 . 9 0}$ \\
\hline
\end{tabular}

Table 9: The natural frequencies ( $\mathrm{rad} / \mathrm{s}$ ) of numerical solutions under different material densities and rise span ratios

\begin{tabular}{cccccccc}
\hline$\kappa_{2}$ & $\rho_{0}\left(\mathrm{~kg} / \mathrm{m}^{2}\right)$ & 0.7 & 1.2 & 1.7 & 2.2 & 2.7 & 3.2 \\
\hline $1 / 10$ & 1st order & 683.61 & 534.07 & $\mathbf{4 4 7 . 6 8}$ & 392.70 & 361.28 & 329.87 \\
& 2nd order & 1107.73 & 872.11 & $\mathbf{7 4 6 . 4 4}$ & 667.90 & 604.76 & 573.34 \\
& 3rd order & 1186.27 & 911.06 & $\mathbf{7 6 9 . 6 9}$ & 691.15 & 628.32 & 603.19 \\
$1 / 12$ & 1st order & 549.78 & 416.26 & $\mathbf{3 6 1 . 2 8}$ & 322.01 & 290.60 & 267.04 \\
& 2nd order & 879.65 & 801.11 & $\mathbf{6 8 3 . 6 1}$ & 636.49 & 581.19 & 541.92 \\
& 3rd order & 1029.19 & 832.52 & $\mathbf{7 0 6 . 8 6}$ & 691.15 & 628.32 & 596.90 \\
\hline
\end{tabular}



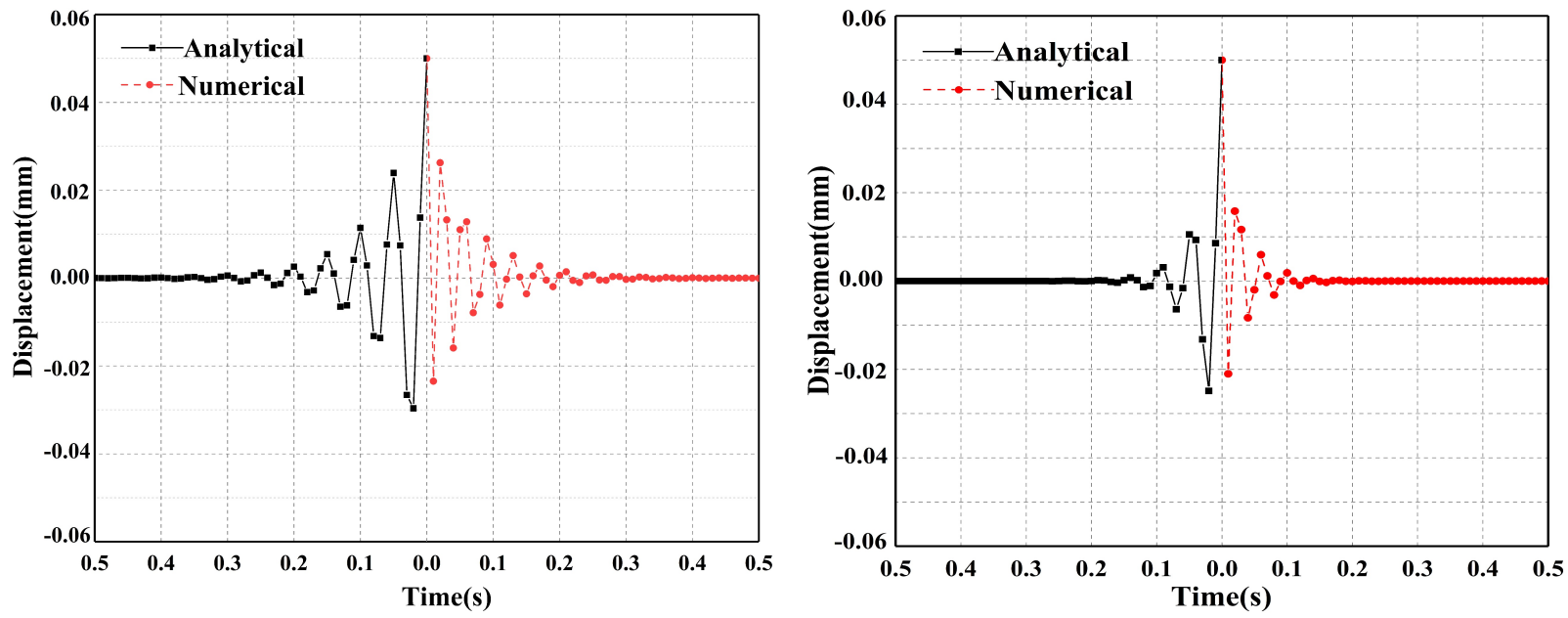

(a) $c=50 \mathrm{Ns} / \mathrm{m}$

(b) $c=100 \mathrm{Ns} / \mathrm{m}$
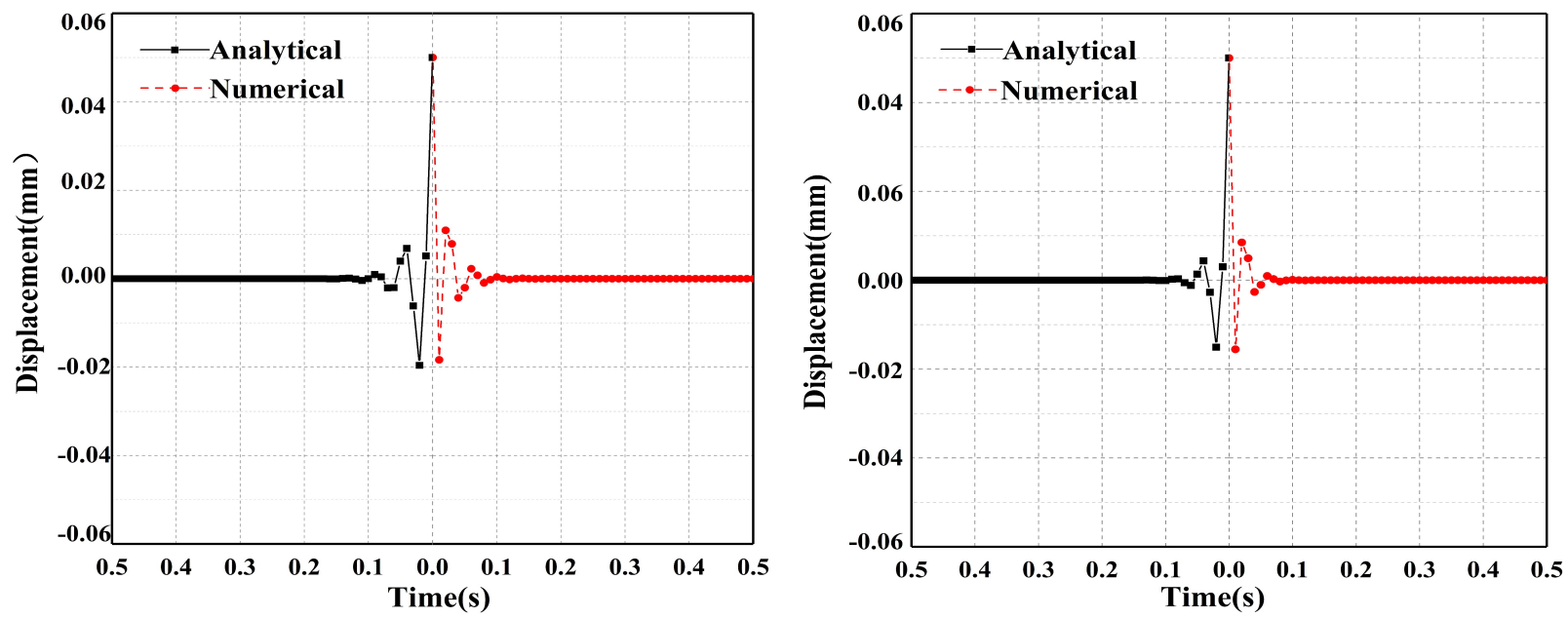

(c) $c=150 \mathrm{Ns} / \mathrm{m}$

(d) $c=200 \mathrm{Ns} / \mathrm{m}$

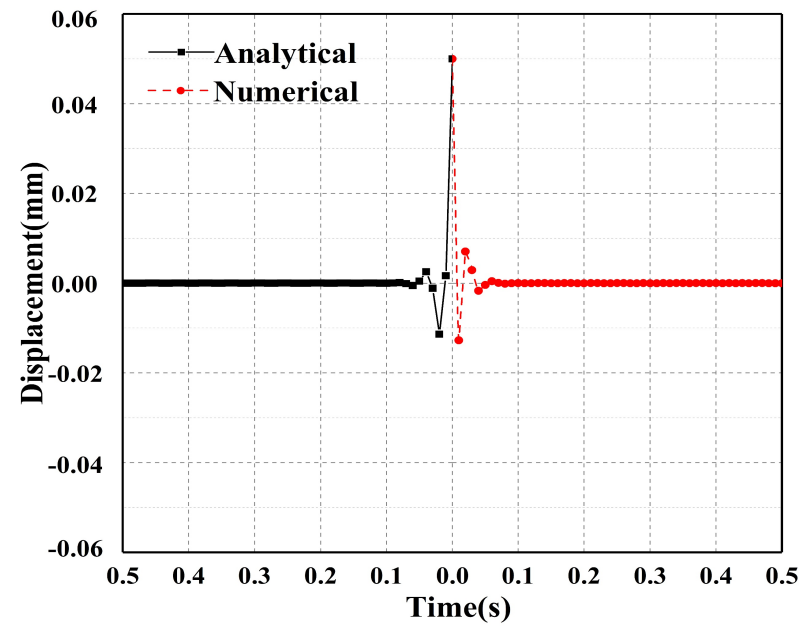

(e) $c=250 \mathrm{Ns} / \mathrm{m}$

Figure 13: Displacement time history curve of the point A under different viscous damping 
(3) By comparing the analytical and numerical results, it can be seen that they are close and the change law is basically the same.

\subsubsection{Effect of material density}

In order to analyze the effect of material density on nonlinear vibration, the first three order natural frequencies of nonlinear vibration under different material densities at rise span ratio $\kappa_{2}=1 / 10$ are calculated respectively. Assuming that the initial displacement of nonlinear vibration is $A=0.05 \mathrm{~m}$ and other material parameters invariant, the calculation results are shown in Tables 8-9.

It can be seen from Tables 8-9 that the natural frequency of nonlinear vibration decreases with the increase of membrane density, and increases with the increase of rise span ratio and order. The natural frequency of the numerical solution is slightly less than that of the analytical solution.

\section{Conclusion}

In this paper, the Galerkin method and the KBM perturbation method were used to solve the damped nonlinear vibration problem of anisotropic prestressed composite fabric membrane structure with skeleton support. The fourth order Runge-Kutta method was used to determine the effective range of the analytical solution, and a numerical example was given to verify it. The effects of membrane material and structure parameters on the nonlinear vibration characteristics of membrane were analyzed. The conclusions are as follows.

(1) Comparing with the numerical solution of RungeKutta, the analytical solution obtained by KBM perturbation method is effective when the initial displacement $A \in[0,0.06] \mathrm{m}$. It can well simulate the nonlinear vibration behavior of the cylindrical membrane structure.

(2) The nonlinear vibration of membrane is affected by the rise span ratio, prestress, elastic modulus ratio, viscous damping, length width ratios and material density.

(3) With the same initial displacement, the natural frequency of nonlinear vibration decreases with the increase of time, increases with the increase of prestress and rise span ratio. When the length of the membrane is larger than the width, the nonlinear vibration frequency of the membrane decreases with the increase of the length width ratio. In the practical engineering, the transverse stiffness of membrane structure can be improved by increasing prestress and rise span ratio, and the dynamic characteristics of nonlinear vibration membrane structure should be fully considered.

(4) Considering the influence of fabric composite membrane parameters on nonlinear vibration, when the elastic modulus in $x$ direction is greater than that in $y$ direction, the nonlinear vibration frequency of membrane increases with the increase of elastic modulus ratio. The attenuation rate of nonlinear vibration increases with the increase of viscous damping, and the natural frequency decreases with the increase of material density. Therefore, the influence of membrane parameters on the nonlinear vibration of membrane should be fully considered in the actual structure.

(5) It can be seen from the calculation of an example that the basic change trend of analytical and numerical calculation is consistent. The research results in this paper can provide a reference for the selection of fabric composite membrane materials, and also provide a theoretical basis for the subsequent research on wind resistance and wind-driven rain dynamic response of skeleton supported fabric composite membrane structures.

Funding information: This work was supported by the Guangdong Basic and Applied Basic Research Foundation (Project number: 2019A1515011063), the National Natural Science Foundation of China (Project Number: 51608060) and Guangzhou Science and technology project (Project number: 202102010455).

Author contribution: Changjiang Liu: Conceptualization, Methodology, Funding acquisition, Writing-Review \& Editing. Mengfei Wang: Validation, Writing-Original Draft, Visualization, Software, Data Curation. Zhoulian Zheng: Resources, Conceptualization, Methodology, Writing-Review \& Editing. Jian Liu: Resources, Conceptualization, Methodology, Funding acquisition. Haibing Xie: Writing-Review \& Editing, Supervision. Su Jiang: Writing-Review \& Editing, Supervision. David Hui: Writing-Review \& Editing, Validation.

Conflict of interest: One of the co-authors (Prof. David Hui) is an Editor in Chief of Reviews on Advanced Materials Science. 
Data availability statement: The data that support the findings of this study are available from the corresponding author, upon reasonable request.

\section{References}

[1] Tang, T., D. H. Yang, L. Wang, J. R. Zhang, and T. H. Yi. Design and application of structural health monitoring system in longspan cable-membrane structure. Earthquake Engineering and Engineering Vibration, Vol. 18, No. 2, 2019, pp. 461-474.

[2] Liu, X., G. P. Cai, F. J. Peng, and H. Zhang. Active control of largeamplitude vibration of a membrane structure. Nonlinear Dynamics, Vol.93, No.2, 2019, pp. 629-642.

[3] Hui, D. Influence of Geometric Imperfections and In-Plane Constraints on Nonlinear Vibrations of Simply Supported Cylindrical Panels. ASME Journal of Applied Mechanics, Vol.51, 1984, pp. 383-390.

[4] Hui, D. Accurate Backbone Curves for a Modified-Duffing Equation for Vibrations of Imperfect Structures with Viscous Damping. ASME Journal of Applied Mechanics, Vol.112, 1990, pp. 304-311.

[5] Awrejcewicz, J., V. A. Krysko, and A. N. Kutsemako. Free vibrations of doubly curved in-plane non-homogeneous shells. Journal of Sound and Vibration, Vol. 225, No. 4, 1999, pp. 701-722.

[6] Krysko, V. A., J. Awrejcewicz, and S. A. Komarov. Nonlinear deformations of spherical panels subjected to transversal load action. Computer Methods in Applied Mechanics and Engineering, Vol.194, No.27-29, 2005, pp. 3108-3126.

[7] Tang, X. F. and J. J. Zhao. Multi-symplectic Runge-Kutta-Nystrom methods for membrance free vibration equation. Journal of $\mathrm{Na}$ ture Scinence of Heilongjiang University, Vol. 27, No. 5, 2010, pp. 603-610.

[8] Xu, Y. P., Z. L. Zheng, C. J. Liu, and W. J. Song. Aerodynamic stability analysis of geometrically nonlinear anisotropy membrane structure with hyperbolic paraboloid. Journal of Engineering Mechanics, Vol. 137, No. 11, 2011, pp. 759-768.

[9] Xu, Y. P., Z. L. Zheng, C. J. Liu, K. Wu, and W. J. Song. Aerodynamic stability analysis of geometrically nonlinear anisotropy membrane structure with hyperbolic paraboloid in sag direction. Wind and Structures, Vol. 26, No. 6, 2018, pp. 355-367.

[10] Liu, C. J., Z. L. Zheng, C. B. Huang, X. T. He, J. J. Sun, and S. L. Chen. The nonlinear instability modes of dished shallow shells under circular line loads. Mathematical Problems in Engineering, Vol. 2011, 2011, id. 793798.

[11] Liu, C. J., Z. L. Zheng, C. B. Huang, W. Qiu, X. T. He, and J. J. Sun. Nonlinear instability of dished shallow shells under uniformly distributed load. International Journal of Structural Stability and Dynamics, Vol. 12, No. 5, 2012, id. 1250035.

[12] Zheng, Z. L., J. J. Guo, W. J. Song, X. T. He, F. Lu, C. X. Xie, et al. Nonlinear free vibration analysis of axisymmetric polar anisotropy circular membranes under the fixed boundary condition. Mathematical Problems in Engineering, (in press), DOI: 10.1155/2014/651356.

[13] Gupta, A. K., N. K. Jain, R. Salhotra, A. M. Rawani, and P. V. Joshi. Effect of fiber orientation on non-linear vibration of partially cracked thin rectangular anisotropy micro plate: An analytical approach. International Journal of Mechanical Sciences, Vol. 105, 2016, pp. 378-397.
[14] Sofiyev, A. H., D. Hui, V. C. Haciyev, H. Erdem, G. Q. Yuan, E. Schnack, et al. The nonlinear vibration of anisotropy functionally graded cylindrical shells surrounded by an elastic foundation within first order shear deformation theory. Composites Part B: Engineering, Vol. 116, 2017, pp. 170 -185.

[15] Sofiyev, A. H. and N. Kuruoglu. Combined effects of transverse shear stresses and nonlinear elastic foundations on the dynamic response of heterogeneous anisotropy cylindrical shells. Composite Structures, Vol. 166, 2017, pp. 153-162.

[16] Li, D., Z. L. Zheng, Y. Tian, J. Y. Sun, X. T. He, and Y. Lu. Stochastic nonlinear vibration and reliability of anisotropy membrane structure under impact load. Thin-Walled Structures, Vol. 119, 2017, pp. 247-255.

[17] Li D., Z. L. Zheng, and M. D. Todd. Nonlinear vibration of anisotropy rectangular membrane structures including modal coupling. Journal of Applied Mechanics, Vol. 85, No. 6, 2018, id. 061004.

[18] Oberst, S. and S. Tuttle. Nonlinear dynamics of thin-walled elastic structures for applications in space. Mechanical Systems and Signal Processing, Vol. 110, 2018, pp. 469-484.

[19] Tang, D. F., C. W. Lim, L. Hong, J. Jiang, and S. K. Lai. Dynamic response and stability analysis with newton harmonic balance method for nonlinear oscillating dielectric elastomer balloons. International Journal of Structural Stability and Dynamics, Vol. 18, No. 12, 2018, id.1850152.

[20] Wu, J. M., M. Y. Shao, Y. Wang, Q. M. Wu, and Z. H. Nie. Nonlinear vibration characteristics and stability of the printing moving membrane. Journal of Low Frequency Noise Vibration and Active Control, Vol. 36, No. 3, 2017, pp. 306-316.

[21] Shao, M. Y., J. M. Wu, Y. Wang, and S. D. Ying. Nonlinear dynamical behaviors of a moving membrane under external excitation. Journal of Low Frequency Noise Vibration and Active Control, Vol. 37, No. 4, 2018, pp. 774-788.

[22] Shao, M. Y., J. M. Wu, Y. Wang, and Q. M. Wu. Nonlinear parametric vibration and chaotic behaviors of an axially accelerating moving membrane. Shock and Vibration, (in press), DOI: $10.1155 / 2019 / 6294814$

[23] Shao, M. Y., J. M. Wu, Y. Wang, H. M. Zhang, and Q. M. Wu. Transverse vibration of a moving viscoelastic hard membrane containing scratches. Mathematical Problems in Engineering, (in press), DOI: $10.1155 / 2019 / 5671361$.

[24] Shao, M.Y., J.M. Wu, Y. Wang, Q.M. Wu, Z. Tian. Nonlinear vibration and stability of a moving printing web with variable density based on the method of multiple scales. Journal of Low Frequency Noise Vibration and Active Control, Vol. 38, No. 3-4, 2019, pp. 1096-1109.

[25] Liu, C. J., M. D. Todd, Z. L. Zheng, and Y. Y. Wu. A nondestructive method for the pretension detection in membrane structures based on nonlinear vibration response to impact. Structure Health Monitoring, Vol. 17, No. 1, 2018, pp. 67-79.

[26] Wang, X. M., J. H. Yuan, and H. R. Zhai. Analysis of bifurcation and chaos of the size-dependent micro-plate considering damage. Nonlinear Engineering, Vol. 8, No. 1, 2019, pp. 461-469.

[27] Awrejcewicz, J. and L. P. Dzyubak. Chaos caused by hysteresis and saturation phenomenon in 2-DOF vibrations of the rotor supported by the magneto-hydrodynamic bearing. International Journal of Bifurcation and Chaos, Vol. 21, No. 10, 2011, pp. 28012823.

[28] Stepanova, L.V. and S. A. Igonin. Perturbation method for solving the nonlinear eigenvalue problem arising from fatigue crack 
growth problem in a damaged medium. Applied Mathematical Modelling, Vol. 38, No. 14, 2014, pp. 3436-3455.

[29] Yazdi, A. A. Assessment of homotopy perturbation method for study the forced nonlinear vibration of anisotropy circular plate on elastic foundation. Latin American Journal of Solids and Structures, Vol. 13, No. 2, 2016, pp. 243-256.

[30] Zhu, C. S., X. Q. Fang, J. X. Liu, and H. Y. Li. Surface energy effect on nonlinear free vibration behavior of anisotropy piezoelectric cylindrical nano-shells. European Journal of Mechanics A-Solids, Vol. 66, 2017, pp. 423-432.

[31] Liu, C. J., X. W. Deng, J. Liu, and Z. L. Zheng. Impact induced nonlinear damped vibration of fabric membrane structure: Theory, analysis, experiment and parametric study. Composites Part B, Vol. 159, 2019, pp. 389-404.

[32] Liu, C. J., F. Wang, J. Liu, X. W. Deng, Z. L. Zhang, and H. B. Xie. Theoretical and numerical studies on damped nonlinear vibration of orthotropic saddle membrane structures excited by hailstone impact load. Shock and Vibration, Vol. 2019, 2019, id. 9234832.

[33] Singh, P. and D. Sharma. Comparative study of homotopy perturbation transformation with homotopy perturbation Elzaki transform method for solving nonlinear fractional PDE. Nonlinear Engineering, Vol. 9, No. 1, 2020, pp. 60-71.

[34] Dong, Y. F., X. F. Yao, H. Yan, L. Yuan, and H. Yang. Macro- and mesoscopic mechanical properties of complex fabric rubber composite under different temperatures. Composite Structures, Vol. 230, 2019, id.111510.

[35] Bolcu, D. and M. M. Stanescu. A study of the mechanical properties of composite materials with a dammar-based hybrid matrix and two types of flax fabric reinforcement. Polymers, Vol. 12, No. 8, 2020, id. 1649.

[36] Lofy, J., V. Gasparian, Z. Gevorkian, and E. Jodar. Faraday and Kerr effects in right and left-handed films and layered materials. Reviews on Advanced Materials Science, Vol.59, No.1, 2020, pp.243-251.

[37] Wang, Y., J. Q. Feng, L. H. Jin, C. S. Li. Photocatalytic reduction of graphene oxide with cuprous oxide film under UV-vis irradiation. Reviews on Advanced Materials Science, Vol. 59, No. 1, 2020, pp. 207-214.

[38] Chang, J. H. Equibiaxially stretchable colorless and transparent polyimides for flexible display substrates. Reviews on Advanced Materials Science, Vol. 59, No. 1, 2020, pp.1-9.

[39] Shi, T. B., J. H. Hu, W. J. Chen, and C. J. Gao. Biaxial tensile behavior and strength of architectural fabric membranes. Polymer Testing, Vol. 82, 2020, DOI: 10.1016/j.polymertesting.2019.106230.

[40] Li, H. C., F. Z. Pang, X. R. Wang, Y. Du, and H. L. Chen. Free vibration analysis for composite laminated doubly-curved shells of revolution by a semi analytical method. Composite Structures, Vol. 201, 2018, pp. 86-111.

[41] Li, H. C., F. Z. Pang, X. H. Mao, S. Y. Gao, and F. Liu. A semi analytical method for free vibration analysis of composite laminated cylindrical and spherical shells with complex boundary conditions. Thin-walled Structures, Vol. 136, 2019, pp. 200-220.
[42] Li, H. C., F. Z. Pang, H. L. Chen, and Y. Du. Vibration analysis of functionally graded porous cylindrical shell with arbitrary boundary restraints by using a semi analytical method. Composites Part B: Engineering, Vol. 164, 2019, pp. 249-264.

[43] Li, H. C., F. Z. Pang, C. Gao, and R. D. Huo. A Jacobi-Ritz method for dynamic analysis of laminated composite shallow shells with general elastic restraints. Composite Structures, Vol. 242, 2020, id. 112091.

[44] Liu, C. J., X. W. Deng, and Z. L. Zheng. Nonlinear wind-induced aerodynamic stability of anisotropy saddle membrane structures. Journal of Wind Engineering and Industrial Aerodynamics, Vol. 164, 2017, pp. 119-127.

[45] Liu, C. J., Z. L. Zheng, L. Jun, J. J. Guo, and K. Wu. Dynamic analysis for nonlinear vibration of prestressed anisotropy membranes with viscous damping. International Journal of Structural Stability and Dynamics, Vol.13, No.2, 2013, id. 1350018.

[46] Liu, C. J., Z. L. Zheng, X. Y. Yang, and H. A. Zhao. Nonlinear Damped Vibration of Pre-Stressed Anisotropy Membrane Structure under Impact Loading. International Journal of Structural Stability and Dynamics, Vol. 14, No. 01, 2014, id.1350055.

[47] Li, D., Z. L. Zheng, C. Y. Liu, G. X. Zhang, Y. S. Lian, Y. Tian, et al. Dynamic response of rectangular prestressed membrane subjected to uniform impact load. Archives of Civil and Mechanical Engineering, Vol. 17, No. 3, 2017, pp. 586-598.

[48] Liu, C. J., J. Feng, Z. L. Zheng, Y. Y. Wu, and J. J. Guo. Nonlinear windinduced instability of orthotropic plane membrane structures. Wind and Structures, Vol. 25, No. 5, 2017, pp. 415-432.

[49] Liu, C. J., Z. L. Zheng, X. T. He, Y. J. Sun, W. J. Song, Y. P. Xu, et al. L$P$ perturbation solution of nonlinear free vibration of prestressed orthotropic membrane in large amplitude. Mathematical Problems in Engineering, Vol. 2010, 2010, id. 561364.

[50] Tang, Y. Q. and T. J. Li Equivalent-force density method as a shapefinding tool for cable-membrane structures. Engineering Structures, Vol. 151, 2017, pp. 11-19.

[51] Liu, C. J., Z. L. Zheng, and X. Y. Yang. Analytical and numerical studies on the nonlinear dynamic response of orthotropic membranes under impact load. Earthquake Engineering and Engineering Vibration, Vol. 15, No. 4, 2016, pp. 657-672.

[52] Liu, C. J., F. Wang, L. He, X. W. Deng, J. Liu, and Y. Y. Wu. Experimental and numerical investigation on dynamic responses of the umbrella membrane structure excited by heavy rainfall. Journal of Vibration and Control, 2020, Early Access, id. 1077546320932691.

[53] Liu, C. J., F. Wang, X. W. Deng, S. Pang, J. Liu, Y. Y. Wu, et al. Hailstone-induced dynamic responses of pretensioned umbrella membrane structure. Advances in Structural Engineering, 2020, Early Access, id. 1369433220940149.

[54] Liu, C. J., X. W. Deng, J. Liu, T. J. Peng, S. P. Yang, and Z. L. Zheng. Dynamic response of saddle membrane structure under hail impact. Engineering Structures, Vol. 214, 2020, id. 110597. 